\title{
Eukaryote-wide sequence analysis of mitochondrial $\beta$-barrel outer membrane proteins
}

Kenichiro Imai ${ }^{1,2}$, Naoya Fujita1,3 ${ }^{1,3}$ Michael Gromiha1', Paul Horton ${ }^{1 *}$

\begin{abstract}
Background: The outer membranes of mitochondria are thought to be homologous to the outer membranes of Gram negative bacteria, which contain 100's of distinct families of $\beta$-barrel membrane proteins (BOMPs) often forming channels for transport of nutrients or drugs. However, only four families of mitochondrial BOMPs (MBOMPs) have been confirmed to date. Although estimates as high as 100 have been made in the past, the number of yet undiscovered MBOMPs is an open question. Fortunately, the recent discovery of a membrane integration signal (the $\beta$-signal) for MBOMPs gave us an opportunity to look for undiscovered MBOMPs.

Results: We present the results of a comprehensive survey of eukaryotic protein sequences intended to identify new MBOMPs. Our search employs recent results on $\beta$-signals as well as structural information and a novel BOMP predictor trained on both bacterial and mitochondrial BOMPs. Our principal finding is circumstantial evidence suggesting that few MBOMPs remain to be discovered, if one assumes that, like known MBOMPs, novel MBOMPs will be monomeric and $\beta$-signal dependent. In addition to this, our analysis of MBOMP homologs reveals some exceptions to the current model of the $\beta$-signal, but confirms its consistent presence in the C-terminal region of MBOMP proteins. We also report a $\beta$-signal independent search for MBOMPs against the yeast and Arabidopsis proteomes. We find no good candidates MBOMPs in yeast but the Arabidopsis results are less conclusive.
\end{abstract}

Conclusions: Our results suggest there are no remaining MBOMPs left to discover in yeast; and if one assumes all MBOMPs are $\beta$-signal dependent, few MBOMP families remain undiscovered in any sequenced organism.

\section{Background}

The outer membrane of mitochondria and chloroplasts as well as Gram-negative bacteria, their evolutionary cousins, harbor proteins of $\beta$-barrel structure, known as $\beta$-Barrel Outer Membrane Proteins (BOMPs). The current version of the endosymbiotic theory of mitochondria origin [1] suggests that mitochondria descend from an $\alpha$-proteobacteria, possibly similar to Rickettsiales, an order of intracellular parasites [2]. BOMPs are predicted to occupy about $3 \%$ of the proteomes of Rickettsiales [3]. Thus, unless Rickettsiales has gained many MBOMPs since its divergence from mitochondria, the $\alpha$-proteobacteria ancestor of mitochondria might be expected to have possessed about 40 BOMPs. Currently four families of mitochodrial BOMPs (MBOMPs) have been identified: Tom40, Sam50 (Tob55), VDAC and Mdm10 [4]. These MBOMPs perform important functions in mitochondria. Tom 40 is

\footnotetext{
* Correspondence: horton-p@aist.go.jp

'AIST, Computational Biology Research Center, Tokyo, Aomi, 135-0064, Japan Full list of author information is available at the end of the article
}

required for the import of mitochondrial precursor proteins into mitochondria, as it forms the import pore of the translocase of the outer membrane (TOM) complex $[5,6]$. Sam50 is the central component of the sorting and assembly machinery (SAM) complex and promotes the integration of proteins into the outer membrane [7-9]. Notably, only Sam50 shows clear sequence homology with non-mitochondrial proteins: Omp85 in bacterial and Toc75-V in chloroplasts [10-12]. Both Tom40 and Sam50 are essential for yeast cell viability $[5,6,9]$. The VDAC family serves as the diffusion pore for small molecules entering or leaving the mitochondria $[13,14]$. VDAC is also thought to contribute to membrane permeability in mitochondrial induced apoptosis $[15,16]$ and is promising as a drug target because of its permeability [17]. In yeast, Mdm10 is required for mitochondrial morphology and dynamics [18]. Homologs of Mdm10 have not been reported in mammals. Mdm10 is reported to be part of the SAM complex and have a role in the biogenesis of MBOMPs $[19,20]$. Recent studies have shown that
C Biomed Central

() 2011 Imai et al; licensee BioMed Central Ltd. This is an Open Access article distributed under the terms of the Creative Commons Attribution License (http://creativecommons.org/licenses/by/2.0), which permits unrestricted use, distribution, and reproduction in any medium, provided the original work is properly cited. 
Mdm10 are members of the ER-mitochondria tethering complex [21].

Based on experimental evidence [22], yeast Mmm2 (Mdm34), was once considered to be an MBOMP, and in our previous work [23] we treated it as such. However, recently pairwise Hidden Markov Model (HMM) comparison has revealed that the SMP domain found in the $\mathrm{N}$-terminal region of Mmm2, belongs to the TULIP superfamily of lipid/hydrophobic ligand-binding domains containing members with known (non-BOMP) structure [24].

As reviewed in [25,26], precursors of MBOMPs are synthesized in the cytosol without a classical $\mathrm{N}$-terminal matrix targeting signal (MTS) or other known targeting signals, but are nonetheless imported across the outer membrane by the TOM complex. In the inter-membrane space, small Tim proteins then escort them to the SAM complex which inserts and assembles them. In spite of significant progress towards the identification of the components involved in the biogenesis of MBOMPs, the import and insertion signal of MBOMPs are still not clear. The first tertiary structure of an MBOMP, human VDAC-1, was recently solved [27-29]. Interestingly, VDAC- 1 consists of 19 transmembrane $\beta$-strands, unlike all known bacterial BOMP (BBOMP) structures, which have even number of $\beta$-strands (but see [30] for an alternative interpretation). There is some sequence similarity between the VDAC family and Tom 40 families. The structure of the C-terminal part of Tom 40 is thought to be similar to VDAC-1 [27,31]. The number of MBOMP families is not known. Many BOMPs have been identified in bacteria, but ony four families have been found to date in mitochondria. Of course, some MBOMP families may remain undiscovered, but it is difficult to experimentally screen for MBOMPs, even in model organisms. Zahedi et al. [32] report a proteomics study of the mitochondrial outer membrane in yeast which detected 112 proteins, including both MBOMPs and $\alpha$-helical proteins. Burri et al. [33] found that 11 proteins precipitate out of a fraction expected to contain MBOMPs and lipid-modified proteins. Neither of these results are specific to MBOMPs.

Searching for new MBOMPs by computational analysis has been difficult as well, due to the extreme divergence in sequence and structure between MBOMPs and their bacterial homologs (or analogs). Recent bioinformatic developments include an analysis using a homology detection method based on transitive sequence similarity search, in which similarity was measured by pairwise HMM profile comparison [34]. In that study the authors identified Sam50, VDAC and Tom 40 as BBOMP homologs, but found no promising novel MBOMPs in the yeast or human proteome.

However, recent experimental studies have provided useful information to search for novel MBOMPs by sequence analysis: the discovery of the $\beta$-signal [35], a $\mathrm{C}$-terminal motif, which is proposed to be the insertion signal of MBOMPs into the outer membrane; the determination of the first tertiary structure of VDAC-1 [27-29]; and the import analysis of BBOMPs in yeast [36]. In particular, the identification of the $\beta$-signal gave us the opportunity to search for novel candidates of MBOMPs by bioinformatics analysis. In preliminary work [23], we used the presence of a conserved $\beta$-signal in mitochondrial proteins to search for new candidate MBOMPs and came to the conclusion that probably very few MBOMPs remain undiscovered.

However, for several reasons, our preliminary study could not be considered conclusive: 1 ) we limited our search to proteins annotated by Uniprot [37] or Gene Ontology [38] as having possible mitochondria localization - but uncharacterized proteins might lack that annotation and 2) we limited the search for $\beta$-signals to the C-terminal 40 residues - but MBOMPs having an internal $\beta$-signal might exist. Moreover, recent experiments expressing BBOMPs in yeast [36], and results we report here on presumed homologs of known MBOMPs, call into question the necessity of the $\beta$-signal for all MBOMPs.

In this paper, we describe an expanded search which addresses those limitations. For this search we developed a new machine learning based predictor, trained on both bacterial and mitochondrial proteins, for the prediction of $\beta$-barrel membrane proteins based on sequence features designed to reflect structural constraints and specific sorting signals that MBOMPs are expected to lack. We report the results of a comprehensive search of known Eukaryotic protein sequences employing our new predictor, conserved $\mathrm{C}$-terminal or internal $\beta$-signals, secondary structure prediction, and literature search. We also use our new predictor to conduct a $\beta$-signal independent search against the yeast and Arabidopsis proteomes. We found no new candidates in yeast, while in Arabidopsis several uncharacterized proteins met the search criteria, and one seems potentially promising.

We conclude that there probably are no new MBOMPs to be found in yeast and maybe very few MBOMPs remaining to be found at all, although we must admit that if $\beta$-signal independent or multimeric MBOMPs exist, our search might miss them.

Since MBOMPs are at the core of all known mitochondria outer membrane channels, our results suggest the possibility that the interface between the mitochondria and the cytosol is largely realized by a handful of protein families.

\section{Results and Discussion Conservation of $\beta$-signal}

Kutik et al. [35] proposed the " $\beta$-signal", with the motif $\mathrm{P}_{\mathrm{o}} \mathrm{xGxxH}_{\mathrm{y}} \mathrm{xH}_{\mathrm{y}}\left(\mathrm{P}_{\mathrm{o}}\right.$, polar residue; $\mathrm{G}$, glycine; $\mathrm{H}_{\mathrm{y}}$, large 
hydrophobic residue; $x$, any residue) occurring near the end of the most $C$-terminal $\beta$-strand. Figure 1 shows the sequence logo $[39,40]$ of 41 and 57 unique $\beta$-signal motif sequences; obtained from multiple sequence alignments of 46 MBOMP homologs with confirmed expression, and 70 MBOMPs including proteins whose existence is only inferred by homology, respectively.

\section{$\beta$-signal position}

For each known MBOMP family (Tom40, Sam50, VDAC and Mdm10), a well conserved $\beta$-signal motif match occurs near the $C$-terminus around or near the final known or predicted $\beta$-strand (Figure 2).

\section{$\beta$-signal consensus}

The increase of available protein sequences since our preliminary study [23], allowed us to refine the $\beta$-signal motif somewhat. As indicated in Table 1 which shows the frequency of amino acid groups in the $\beta$-signal motif match of 70 putative MBOMP homolog sequences, the first residue of the $\beta$-signal motif is usually polar, but no negatively charged residues are observed. The residue following glycine (the 4th position) is almost always hydrophobic. Thus we proposed [23] (and Kutik et al. further clarified [41]) a slightly refined motif pattern: $\mathrm{P}_{\mathrm{o}} \mathrm{xGh} \mathrm{xH}_{\mathrm{y}} \mathrm{xH}_{\mathrm{y}}\left(\mathrm{P}_{\mathrm{o}}\right.$ non-negatively

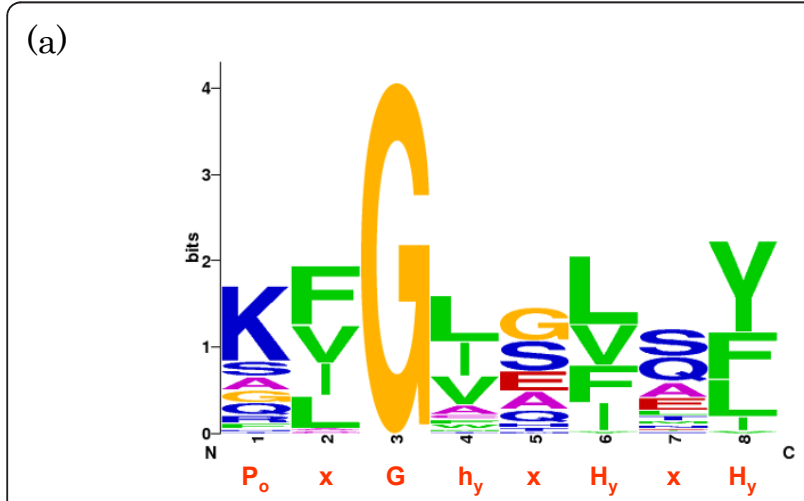

(b)

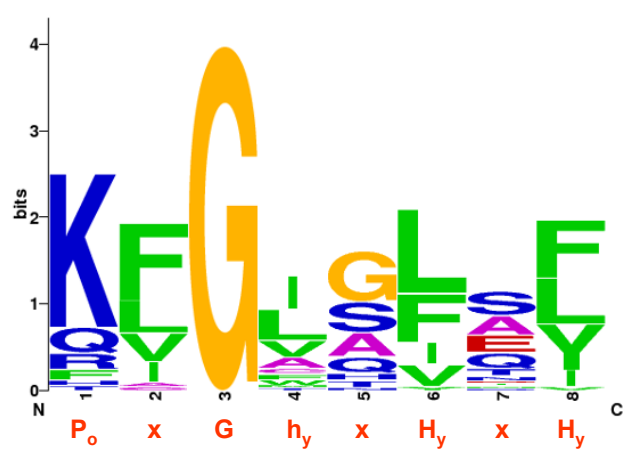

Figure 1 Sequence logos of proposed $\beta$-signal regions of combined ortholog sets. The upper figure is computed from all homologs in Uniprot and the bottom from the subset of those with confirmed expression. charged polar residue, $\mathrm{G}$ glycine, $\mathrm{H}_{\mathrm{y}}$ large hydrophobic residue, $h_{y}$ hydrophobic residue including Ala and Cys, $x$ any residue). As can be seen in Table 1 and the sequence logos of Figure 1, the second position is nearly always a hydrophobic residue. Thus $\mathrm{P}_{\mathrm{o}} \mathrm{H}_{\mathrm{y}} \mathrm{Gh}_{\mathrm{y}} \mathrm{xH}_{\mathrm{y}} \mathrm{xH}$ could also be considered as an alternative consensus sequence. Moreover the other " $x$ " positions also clearly show non-random residue preferences, for example, smaller residues such as glycine, serine, alanine are abundant, however the commonly observed residues do not all share similar properties in terms of hydrophobicity; and because the sequences used are not all mutually independent, the column heights in their sequence logo do not necessarily indicate statistical significance (see methods for details).

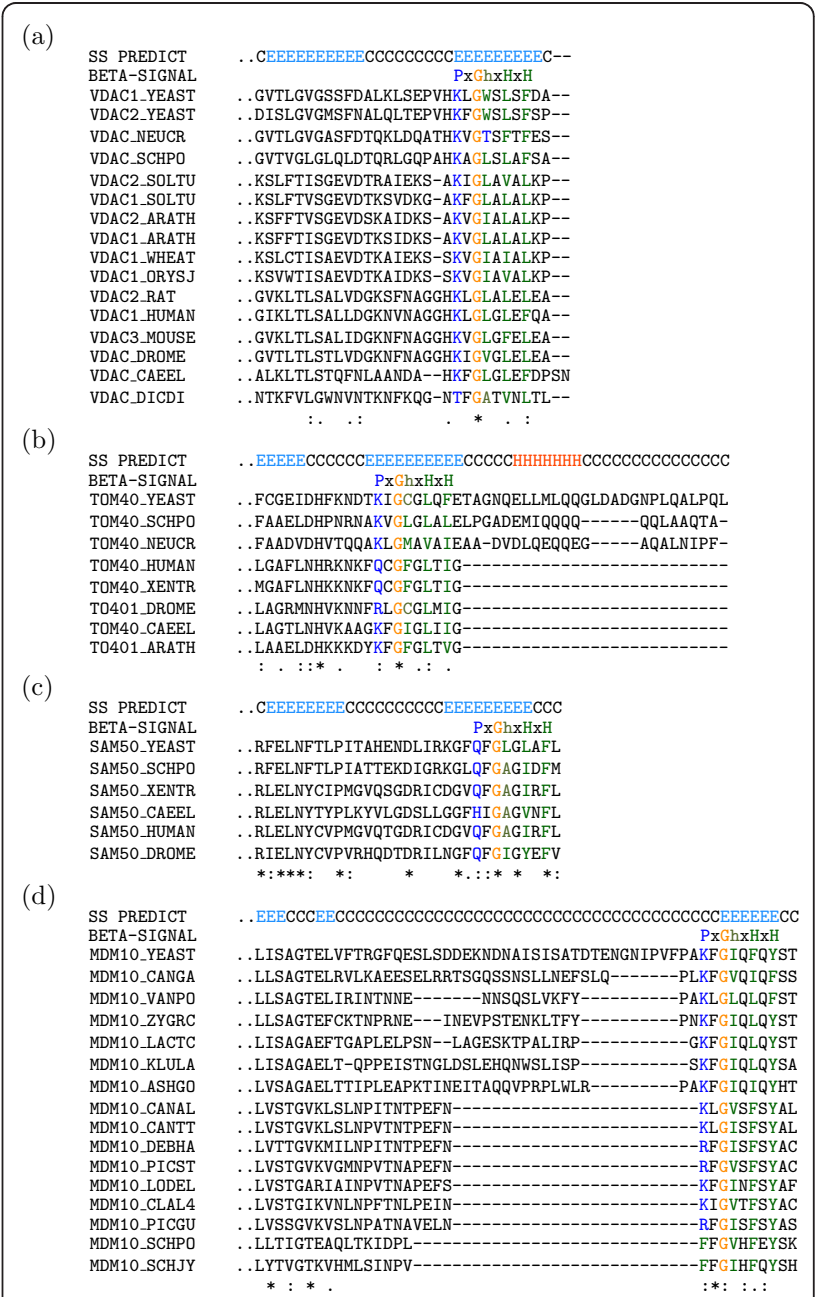

Figure 2 Conserved $\beta$-signals and secondary structure prediction of yeast MBOMPs. In the top track, $\mathrm{H}, \mathrm{E}$, and $\mathrm{C}$

represented $\alpha$-helix, $\beta$-strand and coil, as predicted by PSIPRED or from the experimentally determined structure in the case of VDAC (PDB:2K4T). 
Table 1 The frequency of amino acid groups of $\beta$-signal motif sequences from 70 MBOMP homologs

\begin{tabular}{|c|c|c|c|c|c|c|c|c|c|}
\hline & \multicolumn{9}{|c|}{ Motif position } \\
\hline & Background & $\mathrm{P}_{o}$ & $x$ & G & $\mathrm{h}_{y}$ & $x$ & $\mathrm{H}_{y}$ & $x$ & $\mathrm{H}_{y}$ \\
\hline $\begin{array}{l}\text { Large hydrophobic } \\
(L, I, V, F, M, W, Y)\end{array}$ & 0.32 & 0.03 & 0.96 & 0.00 & 0.89 & 0.00 & 1.00 & 0.16 & 1.00 \\
\hline $\begin{array}{l}\text { Small hydrophobic } \\
\qquad(A, C)\end{array}$ & 0.08 & 0.07 & 0.04 & 0.00 & 0.10 & 0.11 & 0.00 & 0.11 & 0.00 \\
\hline Glycine & 0.07 & 0.16 & 0.00 & 1.00 & 0.00 & 0.24 & 0.00 & 0.00 & 0.00 \\
\hline $\begin{array}{c}\text { Non-negatively charged polar } \\
\text { (K, R, H, S, T, N, Q) }\end{array}$ & 0.39 & 0.74 & 0.00 & 0.00 & 0.01 & 0.50 & 0.00 & 0.61 & 0.00 \\
\hline $\begin{array}{c}\text { Negatively charged } \\
(D, E)\end{array}$ & 0.09 & 0.00 & 0.00 & 0.00 & 0.00 & 0.14 & 0.00 & 0.11 & 0.00 \\
\hline Proline & 0.05 & 0.00 & 0.00 & 0.00 & 0.00 & 0.00 & 0.00 & 0.00 & 0.00 \\
\hline
\end{tabular}

The numbers in parentheses are frequencies of each amino acid group in each position of the $\beta$-signal in 70 MBOMP sequences. "Background" is the overall frequency of each amino acid group in the entire set of sequences.

In any case, the $\beta$-signal region of MBOMPs, for the most part including the " $x$ " positions as well, has a dyad periodicity of hydrophobic residues, and is devoid of the secondary structure breaker proline; as would be expected for a $\beta$-strand in which one side faces the membrane and the other an aqueous pore [42]. This is consistent with the original proposal of Kutik et al. [35] and the recently determined micelle structure of VDAC $[27,28]$, in which the $\beta$-signal occurs in the final $\beta$-strand.

\section{$\beta$-signal motif match occurrence frequency}

The $\beta$-signal was proposed as a signal found near the end of the barrel region, and known MBOMPs generally have $\beta$-signal motif matches near their $\mathrm{C}$-terminal. However, except for the possibly invariant glycine, the $\beta$-signal allows for much variation. Moreover, the $\beta$-signal motif is consistent with the general pattern of alternating hydrophobic and hydrophilic residues expected from any membrane barrel $\beta$-strand. The lack of proline residues is also expected from secondary structure. Thus one may wonder if matches to the $\beta$-signal motif would be expected to occur by chance in most barrel strands. To investigate this we assumed a statistical null model, in which the frequency of $\beta$-signal matches is the same in the length $40 \mathrm{C}$-terminal region as it is in a randomly chosen length 40 region not overlapping with the length 40 C-terminal region. A drawback of this model is that ideally we should exclude the non-barrel portion of MBOMP from consideration but we did not do this. However, the structure of VDAC [27-29] suggests that most of its sequence, at least, is part of its $\beta$-barrel.

In any case, we computed the probability of this null model for VDAC, Tom40, Sam50 and Mdm10 (Table 2), using ten versions of the $\beta$-signal motif. The most significantly enriched pattern for Sam50 is $\mathrm{P}_{\mathrm{o}} \mathrm{H}_{\mathrm{y}} \mathrm{Gh}_{\mathrm{y}} \overline{\mathrm{H}_{\mathrm{y}}} \mathrm{H}_{\mathrm{y}} \mathrm{xH}_{\mathrm{y}}$, where $\overline{H_{y}}$ denotes any residue except [LIVFMWY]. The $\beta$-signal of each of 5 Sam50 homologs matches this pattern, but no other matches are found within those sequence. For VDAC $\mathrm{P}_{\mathrm{o}} \mathrm{H}_{\mathrm{y}} \mathrm{Gh}_{\mathrm{y}} \mathrm{xH}_{\mathrm{y}} \mathrm{xH}_{\mathrm{y}}$ and $\mathrm{P}_{\mathrm{o}} \mathrm{H}_{\mathrm{y}} \mathrm{Gh}_{\mathrm{y}} \overline{\mathrm{H}_{\mathrm{y}}} \mathrm{H}_{\mathrm{y}} \mathrm{xH}$; for Tom40, $\underline{\mathrm{P}_{\mathrm{o}}} \mathrm{xGh}_{\mathrm{y}} \mathrm{xH}_{\mathrm{y}} \mathrm{xH} \mathrm{H}_{\mathrm{y}}$; and for $\mathrm{Mdm} 10, \overline{\mathrm{H}_{\mathrm{y}}} \mathrm{H}_{\mathrm{y}} \mathrm{Gh}_{\mathrm{y}} \mathrm{xH}_{\mathrm{y}} \mathrm{xH}_{\mathrm{y}}$ and $\overline{\mathrm{H}_{\mathrm{y}}} \mathrm{H}_{\mathrm{y}} \mathrm{Gh}_{\mathrm{y}} \overline{\mathrm{H}_{\mathrm{y}}} \mathrm{xH}_{\mathrm{y}}$ are the most enriched. Interestingly, for each of the four MBOMP families, the $\beta$-signal motif is still enriched even if the glycine is ignored. Before seeing this result, we were inclined to view the glycine as the heart of the $\beta$-signal and the other positions to perhaps simply reflecting secondary structure. However, patterns such as $\mathrm{P}_{\mathrm{o}} \mathrm{H}_{\mathrm{y}} \mathrm{xh}_{\mathrm{y}} \overline{\mathrm{H}_{\mathrm{y}}} \mathrm{H}_{\mathrm{y}} \mathrm{xH}$, skipping the requirement for glycine in the third position, still attain $p$-values around $10^{-3}$ or less; suggesting that the other positions may also contribute in a specific way to the recognition of the $\beta$-signal.

Although these results are suggestive, we must warn the reader to interpret the $p$-values with caution. As mentioned above, one problem is the fact that some nonbarrel regions are included in non-C-terminal statistics. Perhaps a more serious flaw is that our logic is somewhat circular, because the $\beta$-signal motif was partially derived from multiple sequence alignment of the $\mathrm{C}$-terminal region of some of these MBOMP homologs (although also due to the results of mutational analysis experiments [35]). Finally the homolog sequences are not mutually independent (maximum pairwise identity is $40 \%$ ), but the $p$-value computation assumes independence.

\section{Conserved $\beta$-signal based MBOMP search}

Our search started with 1,238,639 eukaryotic protein sequences from Uniprot version 15.1. We applied a simple filter to remove nearly identical sequences, clustered with blastclust at $40 \%$ identity and removed singleton clusters to obtain 105,547 homolog clusters. Figure 3 shows our search pipeline (see Methods for more details) and the number of candidates surviving each filter. 16 and 2 protein sequence clusters survived all steps of the C-terminal (C-terminal 40 residues region) and internal $\beta$-signal (all but C-terminal 40 residue region) search respectively.

Table 3 lists the representative proteins for each cluster. In Interpro [43], yeast VDAC and Tom40 are categorized 
Table 2 Comparison of the frequency of $\beta$-signal motif matches in the C-terminal 40 residues compared with the rest of the sequence of MBOMPs

\begin{tabular}{|c|c|c|c|c|}
\hline Motif pattern & VDAC & Tom40 & Sam50 & Mdm10 \\
\hline$P_{0} \times G \times x H_{y} \times H_{y}$ & $\left(4 / 4,0.20,1.6 \times 10^{-3}\right)$ & $\left(5 / 5,0.12,2.5 \times 10^{-5}\right)$ & $\left(5 / 5,0.14,5.4 \times 10^{-5}\right)$ & $\left(6 / 8,0.17,4.9 \times 10^{-4}\right)$ \\
\hline$P_{0} \times G_{y} \times H_{y} \times H_{y}$ & $\left(4 / 4,0.09,6.6 \times 10^{-5}\right)$ & $\left(5 / 5,0.09,5.9 \times 10^{-6}\right)$ & $\left(5 / 5,0.09,5.9 \times 10^{-6}\right)$ & $\left(6 / 8,0.07,2.9 \times 10^{-6}\right)$ \\
\hline $\mathrm{P}_{\mathrm{o}} \mathrm{H}_{\mathrm{y}} \mathrm{G} h_{\mathrm{y}} \times \mathrm{H}_{\mathrm{y}} \times \mathrm{H}_{\mathrm{y}}$ & $\left(4 / 4,0.07,2.4 \times 10^{-5}\right)$ & $\left(4 / 5,0.07,1.1 \times 10^{-4}\right)$ & $\left(5 / 5,0.02,3.2 \times 10^{-9}\right)$ & $\left(4 / 8,0.04,1.6 \times 10^{-4}\right)$ \\
\hline $\mathrm{P}_{\mathrm{o}} \mathrm{H}_{\mathrm{y}} \times \mathrm{h}_{\mathrm{y}} \times \mathrm{H}_{\mathrm{y}} \times \mathrm{H}_{\mathrm{y}}$ & $\left(4 / 4,0.25,3.9 \times 10^{-3}\right)$ & $\left(5 / 5,0.17,1.4 \times 10^{-4}\right)$ & $\left(5 / 5,0.12,2.5 \times 10^{-5}\right)$ & $\left(5 / 8,0.12,1.0 \times 10^{-3}\right)$ \\
\hline $\mathrm{P}_{\mathrm{o}} \mathrm{H}_{\mathrm{y}} \mathrm{Gh}_{\mathrm{y}} \overline{\mathrm{H}_{\mathrm{y}}} \mathrm{H}_{\mathrm{y}} \mathrm{xH}_{\mathrm{y}}$ & $\left(4 / 4,0.07,2.4 \times 10^{-5}\right)$ & $\left(4 / 5,0.07,1.1 \times 10^{-4}\right)$ & $(5 / 5,0.00,0.0)$ & $\left(4 / 8,0.04,1.6 \times 10^{-4}\right)$ \\
\hline $\mathrm{P}_{\mathrm{o}} \mathrm{H}_{\mathrm{y}} \mathrm{xh}_{\mathrm{y}} \overline{\mathrm{H}_{\mathrm{y}}} \mathrm{H}_{\mathrm{y}} \mathrm{xH} \mathrm{H}_{\mathrm{y}}$ & $\left(4 / 4,0.21,1.9 \times 10^{-3}\right)$ & $\left(5 / 5,0.17,1.4 \times 10^{-4}\right)$ & $\left(5 / 5,0.08,3.3 \times 10^{-6}\right)$ & $\left(5 / 8,0.12,1.0 \times 10^{-3}\right)$ \\
\hline$\overline{\mathrm{H}_{\mathrm{y}}} \mathrm{H}_{\mathrm{y}} \mathrm{Gh}_{\mathrm{y}} \mathrm{x} \mathrm{H}_{\mathrm{y}} \mathrm{xH}$ & $\left(4 / 4,0.11,1.5 \times 10^{-4}\right)$ & $\left(4 / 5,0.07,1.1 \times 10^{-4}\right)$ & $\left(5 / 5,0.02,3.2 \times 10^{-9}\right)$ & $\left(7 / 8,0.04,1.3 \times 10^{-9}\right)$ \\
\hline$\overline{\mathrm{H}_{\mathrm{y}}} \mathrm{H}_{\mathrm{y}} \mathrm{xh}_{\mathrm{y}} \times \mathrm{H}_{\mathrm{y}} \mathrm{xH}_{\mathrm{y}}$ & $\left(4 / 4,0.37,1.9 \times 10^{-2}\right)$ & $\left(5 / 5,0.23,6.4 \times 10^{-4}\right)$ & $\left(5 / 5,0.22,5.2 \times 10^{-4}\right)$ & $\left(8 / 8,0.18,1.1 \times 10^{-6}\right)$ \\
\hline$\overline{\mathrm{H}_{\mathrm{y}}} \mathrm{H}_{\mathrm{y}} \mathrm{Gh}_{\mathrm{y}} \times \mathrm{H}_{\mathrm{y}} \mathrm{xH}$ & $\left(4 / 4,0.11,1.5 \times 10^{-4}\right)$ & $\left(4 / 5,0.07,1.1 \times 10^{-4}\right)$ & $(5 / 5,0.00,0.0)$ & $\left(7 / 8,0.04,1.3 \times 10^{-9}\right)$ \\
\hline$\overline{\mathrm{H}_{\mathrm{y}}} \mathrm{H}_{\mathrm{y}} \mathrm{xh}_{\mathrm{y}} \overline{\mathrm{H}_{\mathrm{y}}} \mathrm{H}_{\mathrm{y}} \mathrm{xH}_{\mathrm{y}}$ & $\left(4 / 4,0.33,1.2 \times 10^{-2}\right)$ & $\left(5 / 5,0.23,6.4 \times 10^{-4}\right)$ & $\left(5 / 5,0.20,3.2 \times 10^{-4}\right)$ & $\left(8 / 8,0.16,4.3 \times 10^{-7}\right)$ \\
\hline
\end{tabular}

Statistics on the frequency of matches in the C-terminal versus non-C-terminal part of MBOMPs families is shown for the 4 known MBOMPs and 10 variations of the $\beta$-signal motif. Each cell holds a triple: the fraction of homologs with $\mathrm{C}$-termini which match the motif, the fraction of non-C-terminal length 40 substrings of the MBOMP sequences which match the motif, and a $p$-value. The $p$-value is computed with a binomial test, in which the non-C-terminal frequency (e.g. 0.2 ) is the probability of success and the C-terminal frequency (e.g 4/4) is the observed data.

as Porin, eukaryotic type family and Sam50 is annotated as having the bacterial surface antigen domain. Mdm10 belongs to the MDM10 family in Uniprot. All proteins in the list belong to the same family or share a characteristic domain with known or proposed yeast MBOMPs. Of the $18(16+2)$ surviving representative proteins, we believe each are members of known MBOMP families: 9 Sam50, 4. Tom40, 3 VDAC and 2 Mdm10. The 9 proposed Sam50 homologs are annotated as having a bacterial surface antigen domain in their $\mathrm{C}$-terminal regions, a characteristic shared by the MBOMP Sam50, the

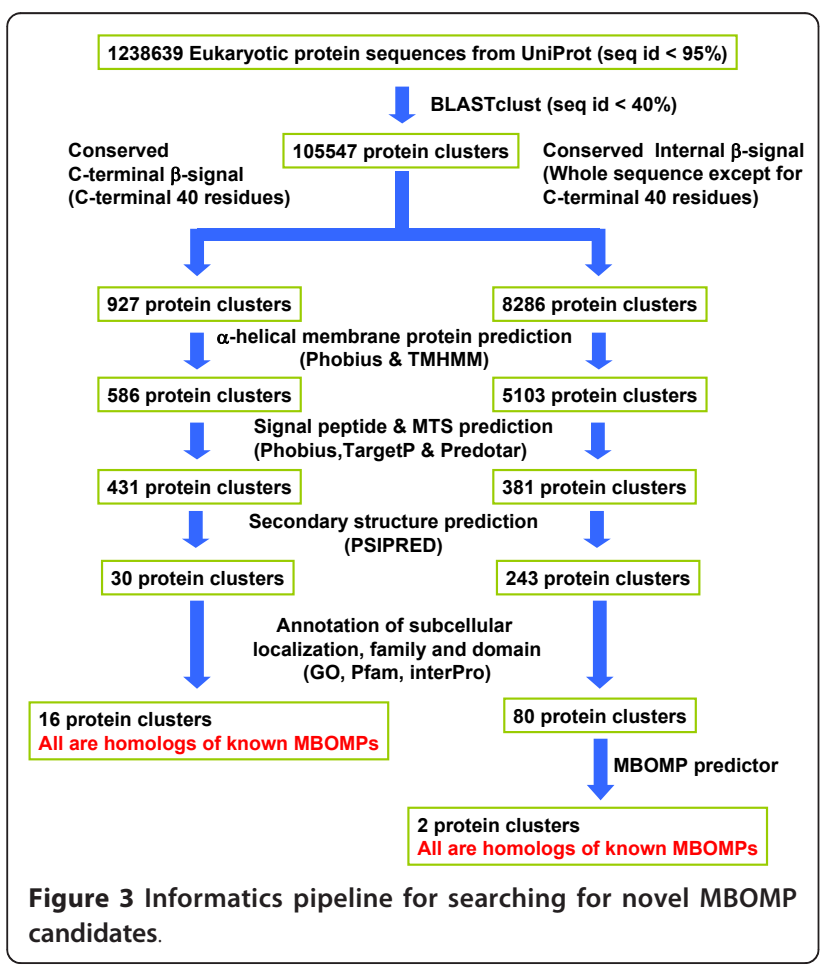

chloroplastic BOMP Toc75-V and bacterial Omp85. An $\mathrm{N}$-terminal polypeptide transport-associated (POTRA) domain is also an important feature of these proteins. The tertiary structure of the POTRA domain of Omp85 revealed that Omp85 of $E$. coli has five POTRA domains which share the fishhook like fold consist of $\beta 1-\alpha 1-\alpha 2$ $\beta 2-\beta 3$, where $\alpha$ and $\beta$ denote $\alpha$ helices and $\beta$ strands (in other bacterial species the number of POTRA domains is not necessarily five but can be between three (or even less) and seven.) $[44,45]$. Sam50 proteins are predicted to have one POTRA domain. Thus we compared the $\mathrm{N}$-terminal region of the 9 proteins by multiple alignment and secondary structure predictions. The multiple alignment and secondary structure prediction suggest that these 9 proteins share one $\beta 1-\alpha 1-\alpha 2-\beta 2-\beta 3$ pattern near their N-terminals (Additional file 1, Figure S1), although there were the differences in the length and composition of the predicted secondary structure elements. Thus we conclude that these 9 proteins are probably Sam 50 homologs. 2 of the 16 representative proteins are the yeast and Lodderomyces elongisporus Mdm10 protein. The remaining 7 representatives are all listed as "Porin, eukaryotic type" in Interpro. One is yeast Tom40. A comparison of secondary structure prediction between yeast VDAC, Tom40, B8NA08, A8I528, B8BQH4, Q293I2 and Q7RE39 indicated that B8NA08, A8I528 and B8BQH4 belong to the VDAC family, while Q293I2 and Q7RE39 probably belong to the Tom40 family (data not shown). Finally, B7Z4T8 shares sequence similarity with Tom40 (Uniprot), although it is much shorter than other Tom40 proteins.

\section{Exceptions to the $\beta$-signal consensus motif}

As detailed above, we believe the two protein sequence clusters which passed the internal $\beta$-signal path of our search pipeline are homologs of known MBOMPs. 
Table 3 List of identified protein clusters with conserved $\beta$-signal

\begin{tabular}{|c|c|c|c|c|c|c|}
\hline Representative protein & $\begin{array}{l}\text { Number of } \\
\text { cluster } \\
\text { member }\end{array}$ & Organism & $\begin{array}{l}\text { Protein } \\
\text { length }\end{array}$ & $\begin{array}{l}\text { Conserved } \\
\text { motif } \\
\text { position }\end{array}$ & $\begin{array}{l}\text { Subcellular } \\
\text { Localization }\end{array}$ & Family and domain \\
\hline \multicolumn{7}{|l|}{ C-terminal $\beta$-signal } \\
\hline SAM50-like protein CG7639 (Q9V784) & 12 & $\begin{array}{c}\text { D. } \\
\text { melanogaster }\end{array}$ & 443 & $435-442$ & Mito OM (G) & $\begin{array}{l}\text { SAM50/omp85 family }(U), \\
\text { Bacterial surface antigen (I) }\end{array}$ \\
\hline $\begin{array}{l}\text { SAM complex subunit of the } \\
\text { mitochondrial outer membrane, putative } \\
\text { (B9WEF8) }\end{array}$ & 6 & C. dubliniensis & 499 & $492-498$ & $\mathrm{OM}(\mathrm{G})$ & Bacterial surface antigen (I) \\
\hline SAM50-like protein (A3LZ83) & 6 & P. stipitis & 489 & $473-480$ & - & - \\
\hline SAM50 (P53969) & 5 & S. cerevisiae & 484 & $476-483$ & Mito OM (U, G) & $\begin{array}{l}\text { SAM50/omp85 family (U), } \\
\text { Bacterial surface antigen (I) }\end{array}$ \\
\hline $\begin{array}{l}\text { SAM50-like protein SpAC17C9.06 } \\
\text { (Q10478) }\end{array}$ & 2 & S. pombe & 475 & $467-474$ & Mito OM (G) & $\begin{array}{l}\text { SAM50/omp85 family }(U), \\
\text { Bacterial surface antigen (I) }\end{array}$ \\
\hline SAM50-like protein gop-3 (P46576) & 2 & C. elegans & 434 & $426-433$ & Mito OM (G) & $\begin{array}{l}\text { SAM50/omp85 family (U), } \\
\text { Bacterial surface antigen (I) }\end{array}$ \\
\hline KLLA0E02223p (Q6CPU1) & 2 & K. lactis & 480 & $472-479$ & $\mathrm{OM}(\mathrm{G})$ & Bacterial surface antigen (I) \\
\hline $\begin{array}{l}\text { Predicted cell surface protein } \\
\text { homologous to bacterial outer } \\
\text { membrane proteins (ISS) (Q017Y3) }\end{array}$ & 2 & O. tauri & 521 & 508-515 & $\mathrm{OM}(\mathrm{G})$ & Bacterial surface antigen (I) \\
\hline TOM40 (P23644) & 36 & S. cerevisiae & 387 & $352-359$ & Mito OM (U, G) & $\begin{array}{l}\text { Tom40 family (U), Porin, } \\
\text { eukaryotic type (I), Mitochondrial } \\
\text { outer membrane translocase } \\
\text { complex, subunit Tom40 (I) }\end{array}$ \\
\hline GA18230 (Q29312) & 8 & $\begin{array}{c}\text { D. } \\
\text { pseudoobscura }\end{array}$ & 321 & $290-297$ & Mito OM (G) & Porin, eukaryotic type (I) \\
\hline $\begin{array}{l}\text { Outer mitochondrial membrane protein } \\
\text { porin (B8NA08) }\end{array}$ & 5 & A. flavus & 346 & $337-344$ & Mito OM (G) & Porin, eukaryotic type (I) \\
\hline $\begin{array}{l}\text { Voltage-dependent anion-selective } \\
\text { channel protein (A81528) }\end{array}$ & 2 & C.reinhardtii & 276 & $268-275$ & $\mathrm{OM}(\mathrm{G})$ & Porin, eukaryotic type (I) \\
\hline Predicted protein (B8BQH4) & 2 & T. pseudonana & 268 & $261-268$ & $\mathrm{OM}(\mathrm{G})$ & Porin, eukaryotic type (I) \\
\hline $\begin{array}{l}\text { cDNA FLJ52528, highly similar to Protein } \\
\text { TOMM40-like (B7Z4T8) }\end{array}$ & 2 & H. sapiens & 210 & $202-209$ & $\mathrm{OM}(\mathrm{G})$ & Porin, eukaryotic type (I) \\
\hline Mdm10 (P18409) & 5 & S. cerevisiae & 493 & $484-491$ & Mito OM (U, G) & $\begin{array}{l}\text { MDM10 family., Protein of } \\
\text { unknown function DUF3722 (I) }\end{array}$ \\
\hline Mdm10 (A5DUG6) & 5 & L. elongisporus & 523 & $496-503$ & Mito OM (U, G) & $\begin{array}{l}\text { MDM10 family., Protein of } \\
\text { unknown function DUF3722 (I) }\end{array}$ \\
\hline
\end{tabular}

Internal $\boldsymbol{\beta}$-signal

\begin{tabular}{|c|c|c|c|c|c|c|}
\hline $\begin{array}{l}\text { Probable mitochondrial import receptor } \\
\text { sub-unit tom40 homolog (Q7RE39) }\end{array}$ & 10 & P. yoelii & 396 & $331-338$ & Mito OM (U, G) & Porin, eukaryotic type (I) \\
\hline $\begin{array}{l}\text { Putative uncharacterized protein } \\
\text { (Q4CQ17) }\end{array}$ & 5 & T. cruzi & 479 & $417-424$ & $\mathrm{OM}(\mathrm{G})$ & Bacterial surface antigen (I) \\
\hline
\end{tabular}

U, G and I in "Subcellular localization" and "Family and domain" represent the source of annotation from Uniprot, Gene ontology and InterPro, respectively.

However it is not clear that they really have internal $\beta$-signals. The $\beta$-signal motif matches of Q7RE39 and Q4CQ17 are not found in their final predicted $\beta$-strand (Figure 4). The proteins Q7RE39, Q4CQ17 and Q293I2 (detected in the $\mathrm{C}$-terminal $\beta$-signal path of our pipeline) have conserved $\beta$-signal motif matches near their fifth, fourth and second predicted $\beta$-strands from their C-terminals, respectively. Focusing on the final predicted $\beta$-strand of these three proteins; Q4CQ17 and Q293I2 have partial $\beta$-signal motif matches such as KFGLTWSS and AFGMRFVV, while Q7RE39 has a match KFGFMMHI but one protein in its cluster, a putative Tom40 of
Plasmodium berghei (Q4Z5G3), only matches (partially) when a gap is inserted after glycine (KFG-MMHI).

Upon examining clusters which did not pass our conserved $\beta$-signal based search, we found some included homologs to known MBOMPs that do not possess perfect matches to the $\beta$-signal motif. Namely, the Tom 40 homolog 2 of Arabidopsis thaliana, Tom40 of Chlamydomonas reinhardtii, and a putative SAM50 homolog of Cryptococcus neoformans (Figure 5). In that figure we also show the N-terminal regions of the BBOMPs: PhoE, OmpA (Escherichia coli) and Omp85 (Neisseria meningitidis) reported by Walther et al. [36] to be 
(a)

SS PREDICT
BETA-SIGNAL
Q7RE39
Q5CF86
B6KRZ3
Q4N6X4
Q4UIN7
A7AQ94
Q4Z5G3
A5K209
B3L7N4
B6AI18

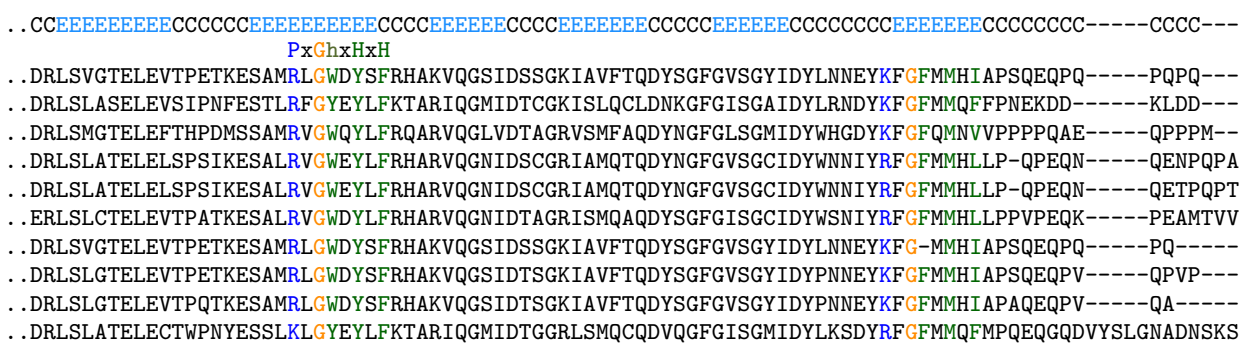

(b)

$\begin{array}{lc}\text { SS PREDICT } & \text {.. CCHHHHHHCCCEEEEEEEEEECCCCCCCCCCEEEEEEEEEEECCCCCCCEEEECCCCCCCCCEEEEEEEECCCCCC } \\ \text { BETA-SIGNAL } & \text { PxGhXHXH } \\ \text { Q4CQ17 } & \text {...DIYQWLRSSASVGFGVVVTRIPLFGVAPSGRFELNFSIPVGIDRSGNVTWRNGNKNLFEHVKFGLTWSSAFSL } \\ \text { A4I4PO } & \text {..KDGYRWLRDCACSVGAGIVITRIPIFGVAPSGRFELNSIPLGIDKQGNIVCRNGPKSLFDRFRFGLVWSFSSSF } \\ \text { Q4FXMO } & \text {..KDSYRWLRDCACSVGAGIVITRIPIFGVAPSGRFELNMSIPLGIDKQGNIVCRNGRKSLFDRFRFGLVWSSNFSF } \\ \text { A4HIY6 } & \text {..KDAYRWLRDCACSIGAGIVITRIPIFGIAPSGRFELNMSIPLGIDKQGNVCRNGRKSLFDRFRFGLVWSSNFSF } \\ \text { Q580K4 } & \text {..FDTYRWFRTCAASVGFGIVVTRVPLFGVTPNGRFELNFCVPVGVDGRGNITWRNGNNRLFDHVKFGLTWSSAFSM }\end{array}$

(c)

$\begin{array}{lc}\text { SS PREDICT } & \text {.. CCCCEEEEEEEEHHCCCCEEEEEEEEECCCCCCCEEEEEEEECCCCCCCC } \\ \text { BETA-SIGNAL } & \text { PxGhXHXH } \\ \text { Q293I2 } & \text {..DSDASVGFMWTKYLNNFPLQMGLSVIMSIPTDRFAFGMRFVVDPSGYRRDN } \\ \text { B4KB83 } & \text {..DSDALVGFMWTKYLTHLPLQMGFSVMSLPTDRFAFGMRFVLDPSGLRRGD } \\ \text { B4M446 } & \text {..DSDALVGFMWTKYLSYIPIQMGFSVVMSLPTDRFAFGMRFVLDPSGLRRGD } \\ \text { B4JFS7 } & \text {..DSDALVGFMWTKYLSHIPLQVGFSVVMNLPINRFAFG IRFVVDPSGLRRGD } \\ \text { B4NA81 } & \text {..DSDASVGFMWTKYLTYLPIQVGFSVVMSMPTNRFAFGTRFVLDPSGLRRGD } \\ \text { B4PRL7 } & \text {.DSDASVGFMWTKYLRHLPLQMGFSVVMNLPTDRFAFGMRFVLDPSGLRRGE } \\ \text { Q9VF44 } & \text {..DSDASVGFMWTKYLTHLPLQMGFSVVMNLPTDRFAFGMRFVLDPSGLRRGE } \\ \text { B3M2Z6 } & \text {..DSDASVGFMWTKYLQHLPLQMGFSVMSLPTDRFAFGMRFVLDPSGLRRGE }\end{array}$

Figure $4 \beta$-Signal matches not always in final predicted $\beta$-strand. Protein clusters which match our automatic criteria of a conserved $\beta$-signal, but with the matches outside of the final predicted $\beta$-signal are shown. The top track indicates predicted $\beta$-strand $(\mathrm{E})$, coil (C), or $\alpha$-helix (H), by PSIPRED. The colored residues occur in full or partial matches to the $\beta$-signal motif.

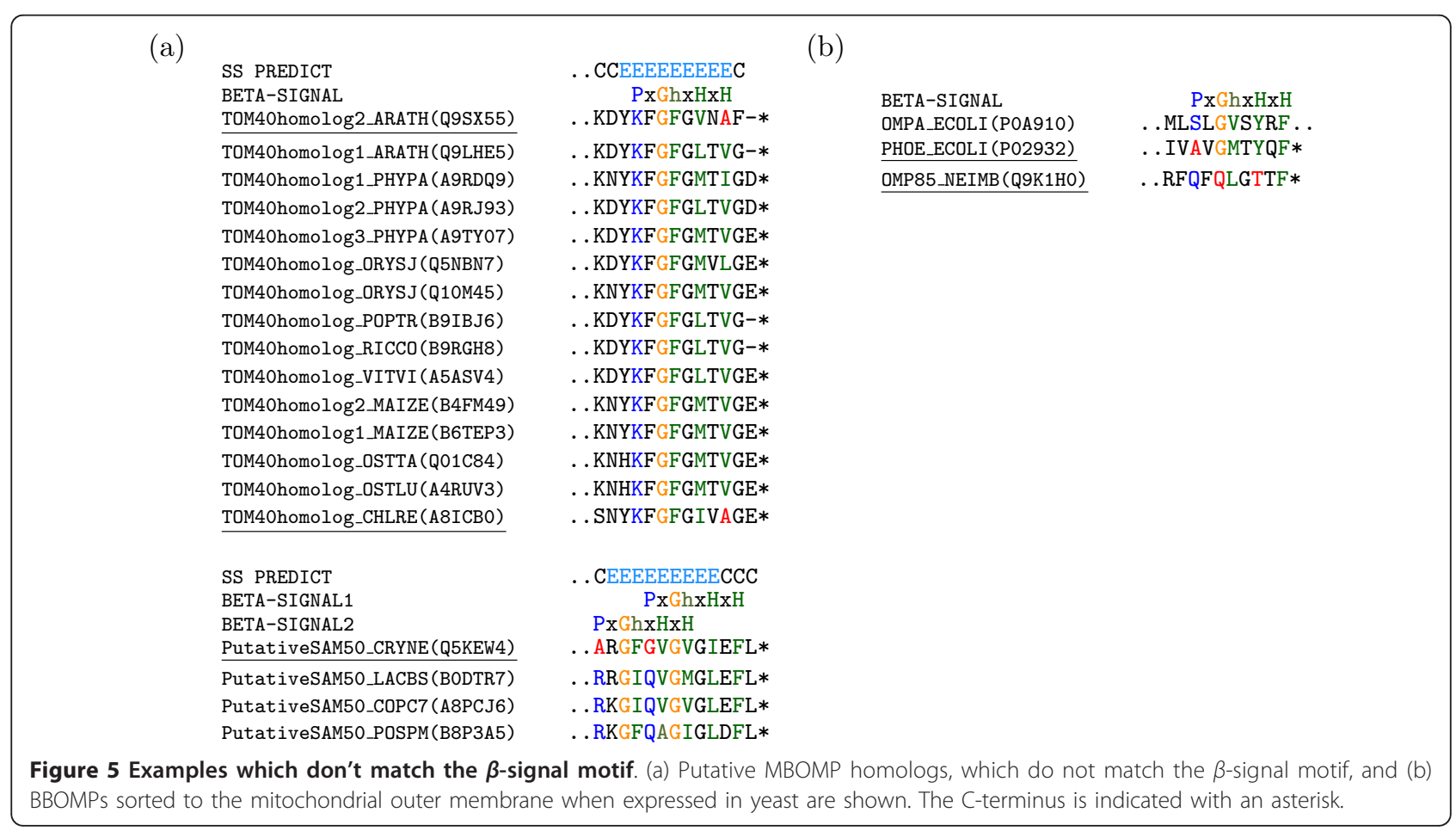


"correctly" integrated into the mitochondrial outer membrane when expressed in yeast. As those authors point out, PhoE and Omp85 do not have an appropriate match to the $\beta$-signal motif (Figure 5).

Moreover, in our analysis of 70 putative known MBOMP homologs, we found that 13 did not match the $\beta$-signal motif. For example, The Neurospora crassa VDAC sequence has threonine in the 4th position following the glycine and the putative Schizosaccharomyces pombe and Schizosaccharomyces japonicus Mdm10 $\beta$-signal sequences are FFGVHFEY and FFGIHFQY, with a phenylalanine instead of a polar residue in the first position (Figure 2). In Uniprot, the protein existence field of 11 of those is listed as "inferred by homology", but two exceptions are verified proteins: VDAC of N.crassa and Mdm10 of S.pombe.

\section{$\beta$-signal independent yeast MBOMP search}

Despite allowing the possibility of internal $\beta$-signals, our conserved $\beta$-signal based search for MBOMPs failed to yield any promising new candidates. To make our search as comprehensive as possible, we also considered the possibility that some MBOMPs have no $\beta$-signal at all.

We searched the yeast proteome (6470 yeast proteins obtained from Uniprot) for MBOMPs lacking $\beta$-signals by combining our new $\beta$-signal independent MBOMP prediction method combined with PSIPRED [46] secondary structure prediction and annotation based manual inspection. The features used by our new MBOMP predictor are: three types of amino acid composition; physicochemical features of the $\mathrm{N}$-terminal sorting region, two amphiphilicity scores based on periodicity and position weight matrices of bacterial transmembrane $\beta$-strands and 2 -strand $\beta$-hairpins (see Figure 6 and Methods for more details). Only a single sequence, that of the uncharacterized protein "YJL217W", passed

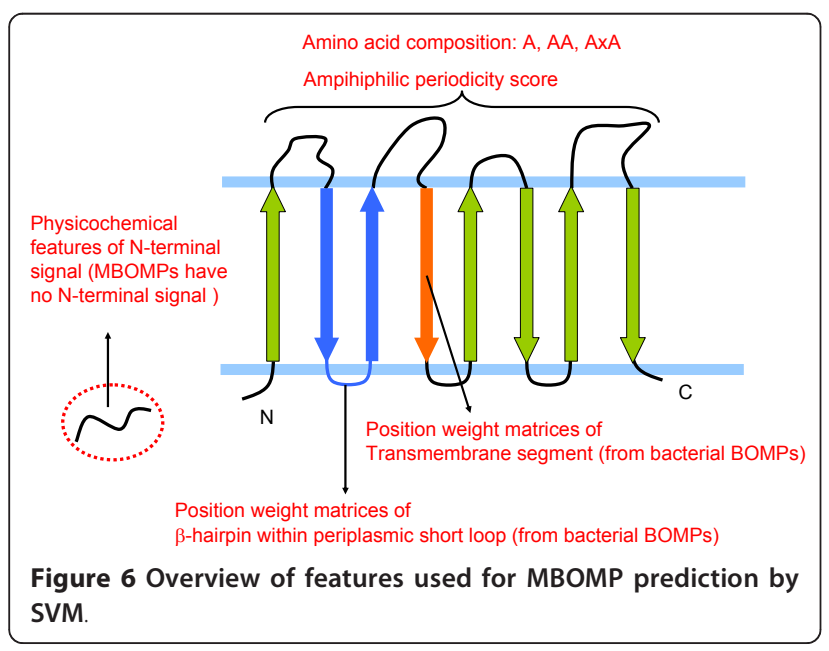

our $\beta$-signal independent search against the yeast proteome.

\section{Comparison to Proteomics Studies in Yeast}

As mentioned in the background section, two experimental studies have tried to identify outer membrane proteins in yeast.

Zahedi et al. [32] report a proteomics study of the mitochondrial outer membrane in yeast which detected 112 proteins including all known MBOMPs. However their method cannot distinguish between MBOMPs, outer membrane $\alpha$-helical proteins (common in mitochondria) and some peripheral membrane proteins. We find only 5 proteins in yeast (including two isoforms of VDAC) instead of 112, but this may be explained by the non-MBOMP proteins included in their list. In another experiment on yeast by Burri et al. [33], 11 proteins precipitated out of a Triton X-114 detergent phase expected to contain MBOMP or lipid-modified proteins. Two were determined to be the MBOMPs VDAC-1 and Tom40, and one, which the authors speculate may be lipid-modified, contained peptides derived by Xdj1. The only information given for the remaining eight is their molecular masses, which are different than known yeast MBOMPs. Without knowing the identity of these proteins we cannot give a specific analysis. One possibility is that those eight proteins are lipid-modified but not MBOMPs.

\section{Is YJL217W an MBOMP?}

Secondary structure prediction shows YJL217W is an all $\beta$-protein with a match to the $\beta$-signal motif around the most C-terminal predicted $\beta$-strand (Additional file 2, Figure S2(a)). (YJL217W was not considered in our conserved $\beta$-signal search because it did not cluster with any other sequences.) YJL217W has no known eukaryotic homologs. According to annotations of InterPro, YJL217W belongs to the DUF1349 family along with several hypothetical bacterial proteins based on sequence similarity; but the function of this family is unknown. YJL217W has no predicted signal peptide or matrix targeting signal, which is consistent with being an MBOMP. However, recently the structure of YJL217W was solved (PDBID:3O12, no published report)(Additional file 2, Figure S2(b)). According to this structure, YJL217W is not a $\beta$-barrel, but instead forms a $\beta$-sandwich structure like Concanavalin. Additionally, YJL217W was not identified as an mitochondrial outer membrane protein in the proteomics study of Zahedi et al. [32], and was found to have a cytosolic localization in a global analysis of protein localization in budding yeast [47]. Thus we do not think YJL217W is an MBOMP.

\section{$\beta$-signal independent Arabidopsis search}

Yeast have relatively small proteomes and are evolutionarily very distant from plants and animals. This limits 
Table 4 List of identified proteins in our Arabidopsis proteome analysis

\begin{tabular}{|c|c|c|c|c|}
\hline Uniprot AC & Identification & Length & Highest score segment (score) & Domain and family in predicted region \\
\hline \multicolumn{5}{|l|}{ MBOMP } \\
\hline Q9SRH5 & VDAC1 & 276 & Whole sequence (0.989) & Porin, eukaryotic \\
\hline Q9SMX3 & VDAC2 & 274 & Whole sequence (1.000) & Porin, eukaryotic \\
\hline Q9FJX3 & - & 276 & Whole sequence (0.989) & Porin, eukaryotic \\
\hline Q9FKM2 & - & 274 & Whole sequence $(0.977)$ & Porin, eukaryotic \\
\hline Q9M2W6 & - & 226 & Whole sequence (0.998) & Porin, eukaryotic \\
\hline Q9FHQ9 & - & 163 & Whole sequence (0.998) & Porin, eukaryotic \\
\hline Q8LGE2 & - & 425 & C-terminal 300 (0.569) & Porin, eukaryotic \\
\hline Q9LHE5 & Tom40 homolog 1 & 309 & Whole sequence (0.991) & Porin, eukaryotic \\
\hline Q9SX55 & Tom40 homolog 2 & 310 & C-terminal 300 (0.999) & Porin, eukaryotic \\
\hline Q8LEH7 & - & 524 & C-terminal $300(0.986)$ & Bacterial surface antigen \\
\hline Q9SRL6 & - & 520 & C-terminal 300 (0.995) & Bacterial surface antigen \\
\hline Q9LXP7 & - & 435 & Whole sequence (0.645) & Bacterial surface antigen \\
\hline Q5PP51 & - & 362 & C-terminal $150(0.900)$ & Bacterial surface antigen \\
\hline \multicolumn{5}{|l|}{ CBOMP } \\
\hline Q9C5J8 & TOC75-V/OEP80 & 732 & C-terminal $300(0.746)$ & Bacterial surface antigen \\
\hline O80565 & OEP37 & 343 & N-terminal $300(0.863)$ & - \\
\hline Q3EBHO & OEP37 homolog & 333 & C-terminal $300(0.891)$ & - \\
\hline Q3EBG9 & OEP37 homolog & 280 & Whole sequence (0.645) & - \\
\hline Q1H5C9 & OEP24 & 213 & Whole sequence (0.562) & - \\
\hline A8MR28 & OEP24 homolog & 167 & Whole sequence (0.666) & - \\
\hline Q9FPG2 & OEP21 & 167 & Whole sequence $(0.925)$ & - \\
\hline Q9LM70 & OEP21 homolog & 203 & C-terminal 150 (0.691) & - \\
\hline Q61D99 & OEP21 homolog & 167 & N-terminal $150(0.875)$ & - \\
\hline \multicolumn{5}{|c|}{ Uncertain protein } \\
\hline Q8VYB6 & - & 491 & Whole sequence $(0.535)$ & Protein of unknown function (DUF1005) \\
\hline Q9LPM5 & - & 460 & C-terminal $450(0.855)$ & Protein of unknown function (DUF1005) \\
\hline Q9MOF0 & - & 424 & $\mathrm{~N}$-terminal $300(0.528)$ & Protein of unknown function (DUF1005) \\
\hline Q9LEU1 & - & 389 & N-terminal $300(0.971)$ & Plant protein of unknown function (DUF868) \\
\hline Q9SIS2 & - & 354 & N-terminal $300(0.655)$ & Plant protein of unknown function (DUF868) \\
\hline Q9M903 & - & 479 & C-terminal $150(0.993)$ & Protein of unknown function (DUF3769) \\
\hline O80503 & - & 451 & C-terminal $300(0.648)$ & Protein of unknown function (DUF3769) \\
\hline Q3EAC5 & - & 134 & Whole sequence $(0.704)$ & Domain of unknown function (DUF3406) \\
\hline$\underline{\text { Q9SXB7 }}$ & - & 424 & C-terminal $300(0.652)$ & - \\
\hline Q9LH72 & - & 483 & C-terminal $300(0.994)$ & - \\
\hline Q9LPG1 & - & 468 & C-terminal 300 (0.816) & - \\
\hline Q8W4R2 & - & 271 & Whole sequence $(0.729)$ & - \\
\hline O48573 & - & 1170 & C-terminal $150(0.625)$ & - \\
\hline Q8VY85 & - & 188 & C-terminal $150(0.608)$ & - \\
\hline Q9LZB6 & - & 177 & C-terminal $150(0.506)$ & - \\
\hline Q9M238 & - & 163 & C-terminal 150 (0.748) & - \\
\hline
\end{tabular}

the scope of what we can conclude from the yeast $\beta$-signal independent search. Ideally we would like to perform a $\beta$-signal independent search on all Eukaryotic proteins, but unfortunately our pipeline partially depends on manual inspection of annotation and ad hoc analysis, so such a search would be infeasible in terms of both the amount of work entailed and the lack of experimental annotation for most species.
As a compromise, we chose to perform a $\beta$-signal independent search on the well annotated plant Arabidopsis. Plants contain mitochondria and chloroplasts, both of which are thought to descend from Gram negative bacteria and are known to have BOMPs in their outer membranes. Our MBOMP predictor was not trained on any chloroplastic BOMPs (CBOMPs), but to the extent that CBOMPs are similar to other 
BOMPs, we might expect our classifier to be able to detect them.

Several CBOMPs have been identified so far (five isoforms (I-V) of Toc75, OEP37, OEP24 and OEP21) $[4,48]$. For Arabidopsis, Uniprot lists three isoforms of Toc75 (III-V), OEP37, OEP24 and OEP21 and four MBOMPs were annotated (two homologs of Tom40 and two of VDAC). We applied our $\beta$-signal independent pipeline to the Arabidopsis proteome (35555 Arabidopsis proteins with less than $90 \%$ sequence identity downloaded from Uniprot). As in the yeast proteome analysis, we used our $\beta$-signal independent MBOMP prediction method combined with secondary structure prediction, $\alpha$-helical membrane protein and signal peptide prediction, and finally manual inspection of annotations to find novel MBOMP (or CBOMP) candidates. 60 proteins passed the automated steps of our pipeline, but we rejected 22 of these based on domain and other annotation (Table 4). 13 of the remaining 38 proteins are annotated BOMPs, 4 MBOMPs (two Tom 40 homolog and two VDAC) and 9 CBOMPs (Toc75-V/OEP80, OEP37, OEP24, OEP21, and five of their homologs). Another five proteins contain the Eukaryotic porin domain and four contain the Bacterial surface antigen (I) domain. This indicates that these proteins probably belong to the VDAC, Tom40, or the Sam50/Omp85 families, respectively. Most of the remaining 16 proteins have no annotation of functional domain or family. We attempted to gain some information by presenting these sequences to the BOMP predictor HHomp [34], and the sequence structure similarity detectors HHpred [49] and FORTE [50]. These predictors suggested that one of the proteins, Q9SXB7:At1g11320, has partial similarity to proteins with $\beta$-barrel structure. However no such similarity was detected for the remaining 15 proteins. Only one of these proteins, Q9M238, posesses an appropriately placed match to the $\beta$-signal, TLGYAFLV (it was not detected in our Eukaryotic-wide search, because its $\beta$-signal was not conserved in all members of its sequence cluster).

In summary we found 13 known or probable MBOMPs from known MBOMP families, 9 known CBOMPs, one promising BOMP candidate and 15 unannotated candidates which are either false positives or distant enough from known BOMPs that they elude state of the art sequence-sequence and sequence-structure similarity detectors. Currently we cannot rule out either possibility. Interestingly, one of these 15 has a $\beta$ signal match, suggesting the possibility that it may be an MBOMP.

Our results differ significantly from an earlier in silico study performed by Schleiff et al. [48], in which 891 candidate CBOMP proteins were identified. The Schleiff et al. pipeline used a BOMP predictor developed by
Wimley [51] and differs from ours in other ways as well, but the overall scheme is very similar. Yet the number of candidate CBOMP proteins differs by an order magnitude. Clearly both estimates cannot be (even approximately) correct, so this discrepency should be explained if possible.

Although we are unable to give a definitive argument in favor of our estimate, we point out one important difference between these two studies. Schleiff et al. [48] used known CBOMPs to tune their pipeline; they lowered the threshold of their $\beta$-barrel predictor score from the value of 1.0 recommended by Wimley based on bacterial BOMPs to 0.7, and made other major adjustments in order to include known CBOMPs. In contrast, our study was originally completely focused on mitochondria and thus all the programs and parameters in our pipeline were chosen without considering information about known CBOMPs.

Despite this fact our pipeline detects 9 out of 11 known CBOMP proteins, which suggests that our results are not overly conservative. However our pipeline did miss two identified CBOMPs (Toc75-III and Toc75-IV) and thus may very well have missed some as yet unidentified CBOMPs.

\section{Is At1g11320 an novel Arabidopsis BOMP?}

Q9SXB7:At1g11320 has 424 residues. The C-terminal 300 residue segment (125-424) was predicted by our predictor to include a $\beta$-barrel. HHomp detected Cyanobacterial SomB as a At1g11320 homolog (probability score $=100 \%$ ), and HHpred and FORTE both judged At1g11320 to be similar to bacterial $\beta$-barrels. HHpred judged the 159-273, 91-232 and 322-424 segments to be similar to the 282-400 segment of $P$. putida toluene transporter TodX (PDBID:3BS0), to 13-141 of E. coli OMPA (PDBID:1QJP) and to 141-253 of $N$. meningitides OpcA (PDBID:2VDF), respectively. While FORTE identified similarities between the 134-424 segment of At1g11320 and the 378-865 segment of $S$. marcescens HasR (PDBID:3SCL).

The interpretation of these results is not completely clear, as the predictions involve a patchwork of different bacterial $\beta$-barrel structures. However, they do suggest the possibility that At1g11320 is a BOMP. It could be either a CBOMP or an MBOMP. The fact that chloroplasts are believed to descend from cyanobacteria and HHomp assigns a high homology probability to a cyanobacterial BOMP, favors the possibility that it is a CBOMP. We also examined the $\mathrm{C}$-terminal region for a $\beta$-signal match. It does have a match, SDGRTIGL, to the originally proposed consensus of $\mathrm{P}_{\mathrm{o}} \mathrm{xGxxH}_{\mathrm{y}} \mathrm{xH}_{\mathrm{y}}$, overlapping the last predicted $\beta$-strand. However, both of the two residues neighboring glycine are hydrophilic and seldom if ever observed in $\beta$-signals (Table 1 ), so most likely this is not a genuine $\beta$-signal. 


\section{Search for novel MBOMPs}

Our comprehensive search based on the $\beta$-signal only detected MBOMPs from known MBOMP families. We also conducted $\beta$-signal independent searches on the yeast and Arabidopsis proteomes; yielding no new candidates for yeast, but a few for Arabidopsis, one of which seems promising. However it is not yet clear whether this candidate really is an MBOMP.

Recently Remmert et al. [34] performed a bioinformatic proteome analysis using a transitive sequence profile similarity based homology detection method, but only detected four known (two VDAC isoforms, Tom40 and Sam50) and no undiscovered MBOMPs in yeast. Subsequently, these authors showed evidence that all outer membrane barrels from Gram-negative bacteria form a monophyletic group that descended from a single $\beta \beta$-hairpin and suggest this probably is true of MBOMPs as well [52]. Their work supports our hypothesis and complements the search we report here. Their work should be very sensitive at detecting homologs, while our novel MBOMP predictor has the potential to detect analogs or distant homologs with no detectable sequence signal remaining.

However it is important to consider what kind of hypothetical MBOMPs could elude our search. Although not designed for CBOMP detection, when we applied our pipeline (without the $\beta$-signal filter) to Arabidopsis it detected most known CBOMPs - but missed two. One of these, Toc75-III, has an N-terminal bipartite transit peptide which is cleaved during biogenesis [4]. Of all known Eukaryotic BOMPs, Toc75-III is the only one with a cleavable targeting signal. If such MBOMPs also exist, our search would probably miss them.

Our search might miss multimeric MBOMPs. Several BBOMPs are known to form a $\beta$-barrel structure from multiple protein chains. For example TolC forms a 12 strand $\beta$-barrel structure from three TolC molecules, each contributing $4 \beta$-strands [53]. Unfortunately TolC, and other multimeric BOMPs, possess large soluble domains in addition to their $\beta$-barrels, and since each sequence only contains a fraction of the $\beta$-barrels, the overall fraction of $\beta$-strand secondary structure is quite small. Our prediction method attempts to account for $\beta$-barrels with soluble domains through the use of segmentation. However this is not sufficient to detect proteins like TolC, for which the $\beta$-barrel sequence region is very short. Currently it is not known if any multimeric MBOMPs exist, but if they do we must admit that our pipeline would probably miss them.

Although the currently accepted known MBOMPs all use the $\beta$-signal for membrane integration, MBOMPs using other mechanisms may exist. If they do, our pipeline would probably miss them. We only applied the $\beta$-signal independent version of our search on two model organisms. Ideally we would like to apply it to all Eukaryotic proteins, but unfortunately that would result in a list of hundreds or thousands of candidate proteins, without much annotation or additional information to cull or prioritize the list. Even when applied to Arabidopsis, our $\beta$-signal independent produced 15 candidate BOMP proteins, for which we can not find corroborating evidence to support or refute. From our results, we can conclude that in the yeast proteome, it is unlikely that we missed any novel undiscovered MBOMP simply because they lack $\beta$-signals, but rather they would have to differ more dramatically from known MBOMPs. Unfortunately we can make no strong conclusions regarding the possibility of $\beta$-signal independent MBOMPs in other species.

Last, but not least, our search would definitely miss any new MBOMPs which only occur in singleton clusters when clustered at $40 \%$ identity. Unfortunately this limitation cannot currently be avoided. As can be seen by the $\beta$-signal independent Aradopsis search, which yielded at least 22 but perhaps as many as 40 false positives, our method depends on an effective $\beta$-signal filter to have enough discriminative power to be applied on a Eukaryote-wide scale. Unfortunately the $\beta$-signal is an information poor motif and occurs quite frequently in non-MBOMP proteins. So the only way to make the $\beta$ signal filter effectively reduce false positives is to require evidence of conservation, and thus we were forced to discard singleton clusters. It is difficult to "prove a negative" and it will always be possible to suggest that an informatics search of the type performed here may miss MBOMPs due to incomplete data or incorrect assumptions. However we believe the search describe here, in conjunction with the consistent results of Remmert et al. [34] obtained with a different methodology, offer strong enough evidence to place the burden of proof on anyone who advocates the existence of undiscovered MBOMP families. Two potential avenues to pursue this are refined proteomics techniques which can distinguish between BOMP and other outer membrane proteins, and sequence analysis of novel organisms not yet included in Uniprot.

\section{Uth1 not an MBOMP?}

We mentioned yeast Uth1 as the only candidate MBOMP identified in our preliminary work [23]. However, further analysis suggests that Uth1 is probably not an MBOMP. Uth1 has a $\beta$-signal motif match near its last predicted $\beta$-strand, however our new $\beta$-signal independent MBOMP predictor does not predict it to be an MBOMP.

Uth1 was reported to have an outer membrane localization [54], but the authors did not specifically claim that it is a $\beta$-barrel. Another study failed to confirm its 
mitochondrial localization, "Preliminary yeast cell fractionation analyses showed that Uth1 with an epitope tag was not efficiently recovered with mitochondria, suggesting that it is not a mitochondrial protein" (Yamano, K. and Endo, T., personal communication). Thus, we conclude that Uth1 probably is not an MBOMP.

\section{$\beta$-signal}

Our MBOMP homolog analysis confirms and slightly refines the $\beta$-signal. As can be inferred from the statistics in Table 1 and 2; in Mdm10 the first position is not always polar, but glycine and other small residues are sometimes seen. From Table 1 we can also see that in the homologs we examined, the fifth position never contains a large hydrophobic residue. From the statistics of Table 1 two patterns with good coverage and $p$-values are $\overline{\mathrm{H}_{\mathrm{y}}} \mathrm{H}_{\mathrm{y}} \mathrm{Gh}_{\mathrm{y}} \mathrm{xH}_{\mathrm{y}} \mathrm{xH}_{\mathrm{y}}$ and $\overline{\mathrm{H}_{\mathrm{y}}} \mathrm{H}_{\mathrm{y}} \mathrm{Gh}_{\mathrm{y}} \overline{\mathrm{H}_{\mathrm{y}}} \mathrm{H}_{\mathrm{y}} \mathrm{xH}$. The $p$-values for these two patterns are nearly the same for the background sequence regions from MBOMP proteins used to compute them. However we would expect the more specific pattern $\overline{\mathrm{H}_{\mathrm{y}}} \mathrm{H}_{\mathrm{y}} \mathrm{Gh}_{\mathrm{y}} \overline{\mathrm{H}_{\mathrm{y}}} \mathrm{H}_{\mathrm{y}} \mathrm{xH}_{\mathrm{y}}$ to discriminate better when non $\beta$-barrel sequences are included in the background. In that case a requirement of no prolines could also be considered as an extra filter, or alternatively a position weight matrix covering these positions could be defined.

Amongst the putative MBOMP homologs, we found 13 exceptions to the core $\beta$-signal consensus, and 12 of them were in $\mathrm{Mdm} 10$. Mdm10 also differs from the other MBOMP families in that it has no known homologs in animals.

Even the exceptions contain the apparently invariant glycine and exhibit the length 2 periodicity expected in a $\beta$-strand. However, our stastical analysis of the frequency of $\beta$-signal motifs in the $\mathrm{C}$-terminal versus other parts of MBOMP sequences (Table 2) suggest that the non-glycine positions of the $\beta$-signal may contribute to its recognition in a more specific way than just by providing an appropriate secondary structure context.

Recent experimental work suggests that the $\beta$-signal is not necessary for mitochondrial outer membrane integration. Walther et al. [36] reported that, when expressed in yeast, some BBOMPs were sorted to the mitochondrial outer membrane. In particular the C-terminus of OMP85_NEIMB does not even have a near match to the $\beta$-signal (Figure 5). It remains to be seen if any naturally occurring MBOMPs lack a $\beta$-signal. As discussed above, in a Eukaryote-wide search, removing the $\beta$-signal filter leads to an unmanageable number of candidates. One idea for future work, would be to use a bacterial-type C-terminal phenylalanine integration signal motif [55] as an alternative filter.

\section{Conclusions}

We developed a novel MBOMP predictor, and used it to perform a Eukaryotic-wide search for $\beta$-signal dependent MBOMPs, and a $\beta$-signal independent search in yeast and Arabidopsis. In the Eukaryotic-wide search we found no promising new MBOMP candidates. Likewise in the yeast $\beta$-signal independent search we found no promising new MBOMP candidates. The results of the $\beta$-signal independent Arabidopsis search were less conclusive. We found one potentially promising new BOMP candidate and 15 more candidate BOMPs which do not appear to have sequence or structural similarities to known BOMPs. We could not find corroborating evidence to support these candidates, so their confirmation or refutation must await further work.

We conclude that the currently identified MBOMP protein families may represent a nearly complete repertoire of $\beta$-signal dependent MBOMPs. If many undiscovered MBOMP families remain in the current sequence databases, they most likely differ from known MBOMPs significantly in terms of structure or biogenesis.

\section{Methods}

\section{Known MBOMP ortholog sets}

Using Swiss-Prot 57.8 [56], we gathered all complete protein sequences with at least $40 \%$ identity to known MBOMPs, a level which strongly suggests similar structure. After removing nearly identical sequence pairs we obtained a set of 70 proteins in which each pair shares less than $90 \%$ sequence identity. The expression of 46 (VDAC:16, Tom40:8, Sam50:6 and Mdm10:16) of these has been confirmed at the protein or transcript level.

\section{Search for novel MBOMPs in eukaryotic proteomes Eukaryotic Protein Sequence Cluster Dataset}

We downloaded all eukaryotic protein sequences in Uniprot Version 15.1 and applied a simple filter to obtain 1,238,639 protein sequences with no more than $95 \%$ sequence identity. We then clustered these sequences at $40 \%$ identity with BLASTClust http://blast. ncbi.nlm.nih.gov/ and removed singleton clusters from further consideration. In this way we obtained 105,547 non-singleton clusters, containing a median of 3 and average of 8.8 sequences per cluster.

We computed a multiple alignment of each cluster with ClustalW [57]. Given the $40 \%$ sequence identity level within clusters, we expect the sequences should be relatively easy to align correctly.

\section{Search Procedure}

We searched intensively for novel MBOMPs, relaxing the constraint imposed in our earlier work, of requiring a $C$-terminal location of potential $\beta$-signals. Figure 3 
shows the pipeline we employed to identify proteins with either $\mathrm{C}$-terminal (C-terminal 40 residues region) or internal (whole sequence except for the C-terminal 40 residues) potential $\beta$-signals. Where a potential $\beta$-signal is a match to the $\beta$-signal motif in each sequence in a cluster, which aligns perfectly in their multiple sequence alignment.

The pipeline is comprised of 6 filters:

- Potential $\beta$-signal: a sequence passes this test if it has an appropriately placed match to the $\beta$-signal motif $\left(\mathrm{P}_{\mathrm{o}} \mathrm{xGh}_{\mathrm{y}} \mathrm{xH}_{\mathrm{y}} \mathrm{xH}_{\mathrm{y}}\right)$ which aligns perfectly in its cluster multiple alignment.

- Not an $\alpha$-helical membrane protein: a sequence fails this test if it is predicted to be an $\alpha$-helical membrane protein by either Phobius [58] or TMHMM [59].

- Not a secretory path protein: a sequence fails this test if it is predicted to have a signal peptide by either TargetP or Phobius.

- No MTS: a sequence fails this test if it is predicted to have a mitochondrial matrix targeting signal (MTS) by either TargetP [60] or Predotar [61].

- Not an $\alpha$-rich protein: a sequence fails this test if it is predicted by PSIPRED [46] to have less than $25 \% \beta$-strand and more than $10 \% \alpha$-helical secondary structure content in the (up to 300 ) residues preceding the $\beta$-signal.

- $\beta$-signal match not of $\alpha$-helical structure: fails test if more than $50 \%$ of the 8 residues of $\beta$-signal match are predicted as $\alpha$-helical structure by PSIPRED.

- Manual inspection: annotation of subcellular localization, family and domain by Gene ontology, Pfam [62] and interPro [43].

- MBOMP predictor: fails test if not predicted to be an MBOMP by our MBOMP predictor (not applied to $\mathrm{C}$-terminal $\beta$-motif matches).

\section{MBOMP Predictor}

We trained a novel MBOMP predictor on known MBOMP sequences, but with some features derived from BBOMP structures. We chose not to use existing tools because they are trained only on bacterial sequences. Instead we trained our MBOMP predictor on a mixture of bacterial and mitochondrial proteins. The features used by our MBOMP predictor are: three types of amino acid composition (standard, bigram and gapped bigram); physicochemical features of the $\mathrm{N}$-terminal sorting region, periodic amphiphilicity scores and position weight matrices (computed from BBOMP structures) to model transmembrane $\beta$-strands and 2-strand $\beta \beta$-hairpins (Figure 6). For a classifier, we chose a support vector machine (SVM) with an RBF kernel, as implemented in the LIBSVM package http://www.csie.ntu.edu.tw/ cjlin/libsvm. All features were linearly scaled to the range $[-1,1]$ before being presented to the SVM classifier.

\section{Features}

Length We do not use length as a feature per se, but we require candidate MBOMPs to be at least 90 residues. This should not exclude single chain barrels, as even the most compact barrels span about 150 residues [63].

Amino Acid Composition It is well known that protein secondary structure correlates with amino acid composition and invokes correlation between nearby residues [64]. Therefore we adopted amino acid composition, dipeptide composition and "skip one dipeptide composition", defined as the frequency of the 400 patterns $A-X-B$, where $X$ is any residue and $A$ and $B$ are fixed amino acid residues, to give a total of 820 features.

Signal Peptide Related Features MBOMPs are not expected to have secretory pathway signal peptides. We therefore included four signal peptide related features. Following the classical model of signal peptides [65], we defined features according a contiguous block of 30 residues, partitioned into the n-, c-, and h-regions, of length 5, 20 and 5 residues respectively (Figure 7). Denoting the Kyte-Doolittle hydrophobicity [66] of the $i$ th residue as $h(i)$, we compute a weighted average of the hydrophobicity near residue $j$ as:

$$
H(j)=\sum_{i=j-14}^{j+14}(15-|i-j|) * h(i) / 15^{2}
$$

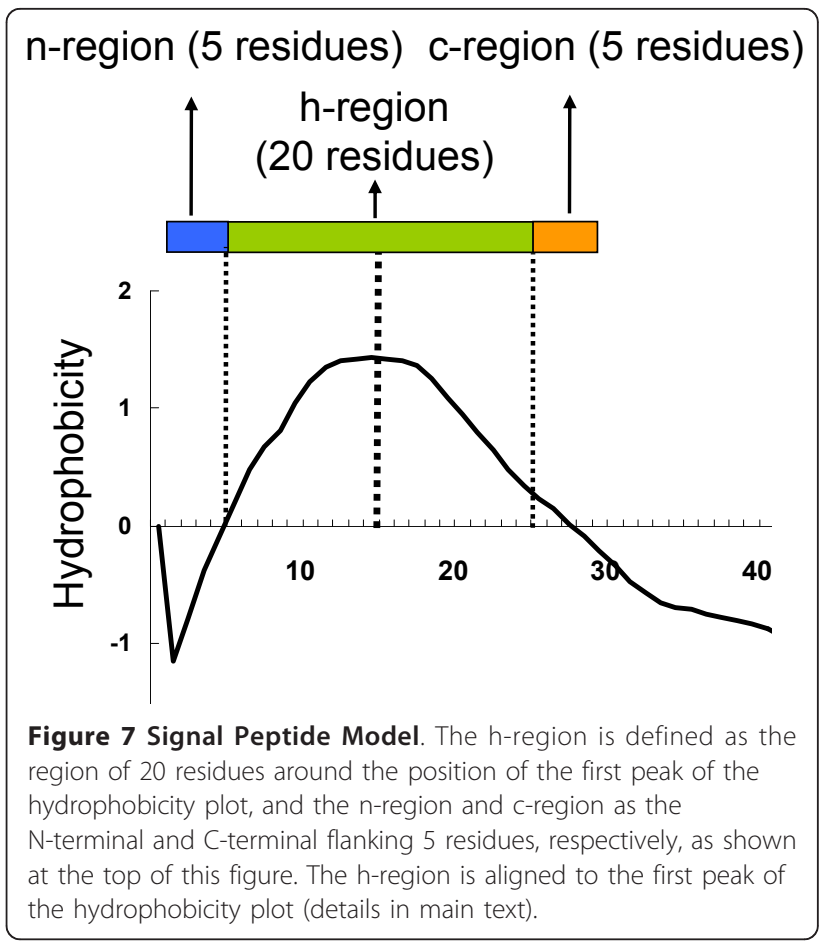


We define the $\mathrm{N}$-terminal peak position as the first peak attaining at least 0.6 , up to the first 70 residues, or the maximum if no such peak exists. More precisely the peak position is arg $\max _{j \in R} H(j)$, where $R$ is either the first contiguous set of positions in the interval $[1,70]$ such that $H(j) \geq 0.6$; or if that set is empty, $R$ is simply the first 70 positions.

When computing features, we aligned the 10th position of the h-region to the $\mathrm{N}$-terminal peak. We used the following features:

$$
\begin{aligned}
& \text { 1) } \sum_{i \in \text { h-region }} h(i), \text { 2) } \sum_{i \in \text { n-region }} p(i), \text { 3) } \sum_{i \in \text { n-region }} n(i) \text {, } \\
& \text { 4) } \sum_{i \in \text { n-region }} n(i) \text { and 5) } \sum_{i \in \text { c-region }} o(i)
\end{aligned}
$$

where $p(i), n(i)$ and $o(i)$ are $0-1$ binary variables equal to 1 when the $i$ th residue is positively $\{K, R, H\}$ or negatively $\{\mathrm{D}, \mathrm{E}\}$ charged, or one of $\{\mathrm{A}, \mathrm{G}, \mathrm{S}\}$, respectively.

Amphiphilicity scores Since every other residue in a BOMP $\beta$-strand tends to be hydrophobic, we define a tile amphiphilicity score:

$$
A(j, l)=\left|\sum_{i \in\{0,2, \ldots l\}} h(j+i)-\sum_{i \in\{1,3, \ldots l-1\}} h(j+i)\right|
$$

for the length $l$ tile starting at position $j$.

Let $A(R, l)$ denote the 60th percentile of $A(j, l)$ for $j$ in sequence region $R$, and $n$ denote the entire sequence length. To account for the fact that BOMPs contain a significant number of residues in loops and often nonbarrel domains as well, we defined three types of region amphiphilicity scores: global, $A(R, l)$ where $R=[1, n]$; N-terminal, $R=\max _{Q \epsilon\{[1,90],[1,91], \ldots[1, n]\}} A(Q, l)$; and Cterminal,

$R=\max _{Q \epsilon\{[n-89, n],[n-90, n], \ldots[1, n]\}} A(Q, l)$. The N- and Cterminal features are designed to detect proteins with $\mathrm{N}$ - or C-terminal barrel domains respectively. Finally we define 9 amphiphilicity related features to show to the classifier: each of the three region amphiphilicity scores computed with tile lengths of 8, 10 and 12 .

PWMs for transmembrane $\beta$-strands and $\beta \beta$-hairpins We collected 42 BBOMP structures with less than $40 \%$ sequence identity from PDB [67]. From these structures we extracted 428 transmembrane segments of lengths 8 , 9 and 10; and $181 \beta \beta$-hairpins (periplasmic short loop plus 8 residues on both sides of the loop) with loop lengths of 3,4 and 5 . As extracted, the $\beta$-strands contained in these segments were not always in phase with each other in terms of amphiphilicity. Thus we first expressed each segment as a vector of hydrophobicity values $h(i)$, and used those vectors to divide each
Table 5 Prediction performance of our SVM MBOMP predictor

\begin{tabular}{ccccccccc}
\hline Fold & TP & FP & FN & TN & Precision & Recall & Specificity & F-measure \\
\hline 1 & 15 & 1 & 4 & 527 & 0.938 & 0.789 & 0.998 & 0.857 \\
2 & 16 & 0 & 3 & 528 & 1.000 & 0.789 & 1.000 & 0.914 \\
3 & 15 & 0 & 4 & 528 & 1.000 & 0.789 & 1.000 & 0.882 \\
4 & 16 & 0 & 3 & 528 & 1.000 & 0.842 & 1.000 & 0.914 \\
5 & 17 & 0 & 1 & 528 & 1.000 & 0.944 & 1.000 & 0.971 \\
Mean & - & - & - & - & 0.988 & 0.842 & 1.000 & 0.908 \\
\hline
\end{tabular}

segment into one of two clusters using the hierarchical clustering function of the $\mathrm{R}$ programming language with Euclidean distance. For the hairpin types we defined a PWM for each cluster, yielding 2 PWMs each, of lengths 19, 20 and 21. The initial PWMs derived from each pair of clusters of the single strand transmembrane segments were nearly identical except for being shifted by one position relative to each other. Thus we combined these PWMs by shifting one of them by one position relative to the other and discarding the unmatched columns at each end. This yielded 3 PWMs of lengths 7, 8 and 9, respectively. For a background model we used the overall amino acid composition of sequences in our dataset.

To convert a PWM $P$, into a numerical feature, we first compute $r$, the maximum log likelihood ratio score between $P$ and the background model for each possible position in the query sequence. More precisely

$$
\begin{gathered}
r=\max _{j=1}^{n-w+1} r(j) \\
r(j)=\sum_{i=1}^{w} \frac{P(s[i+j-1], i)}{B(s[i+j-1]}
\end{gathered}
$$

Where $w$ denotes the width of the PWM, and $s[i]$ the $i$ th character in the query sequence. In general $r$ will tend to increase with sequence length and we speculate that it will follow an extreme value distribution similar to the BLAST statistics [68]. Using non-mitochondrial sequences, we empirically confirmed that the following normalization effectively removes the dependence on sequence length for background sequences (results not shown).

$$
r^{\prime}=r-a^{*} \log (n)
$$

where $a$ is an empirically adjusted constant which differs for each PWM. Thus we defined the nine PWM based features with that formula.

\section{MBOMP Predictor Dataset}

Our dataset contains 94 positive examples, consisting of the known yeast MBOMPs and their presumed (based on sequence similarity and conservation of the $\beta$-signal) 
homologs in other species. No positive example shares more than $40 \%$ identity with any other. For negative examples we used 2640 yeast proteins with clear annotation of subcellular localization of Uniprot and less that $25 \%$ mutual sequence identity.

\section{MBOMP Predictor Results}

To assess the accuracy of our MBOMP predictor, we measured the performance with 5 -fold cross validation. We obtained a precision of $98.8 \pm 0.03 \%$, recall of $84.2 \pm 0.06 \%$ and F-measure of $0.91 \pm 0.04 \%$ (Table 5). To further test the ability of the predictor to generalize to novel MBOMP families, we trained it using only homologs of $\{$ VDAC, Tom40, Sam50\} as MBOMP positive examples (BBOMPs were also included as positive examples in all cases), and then tested whether this predictor could correctly classify Mdm10 (as positive) and Mmm2 family (as negative). We believe this is a good test, because Mdm10 is a bit different from the other MBOMPs (it has not been found in animals and its $\beta$ signal seems a bit divergent), while $\mathrm{Mmm} 2$ is plausible enough as an MBOMP that it was considered to be one for some time. In this test, our predictor predicted nine of twelve Mdm10 homologs and zero of eleven Mmm2 homologs.

\section{$\beta$-signal independent search in yeast and Arabidopsis proteomes}

We searched for MBOMPs lacking $\beta$-signals in $6470 \mathrm{~S}$. cerevisiae and $35555 A$. thaliana proteins found in Uniprot by combining our MBOMP predictor with PSIPRED secondary structure prediction, Phobius $\alpha$ helical membrane protein and signal peptide prediction and finally manual inspection based on annotation. In this search, in addition to whole sequences, we considered $\mathrm{N}$-terminal and $\mathrm{C}$-terminal protein segments of length 150, 300 and 450 residues, to take into account the possibility of multi-domain MBOMPs such as Sam50 (Additional file 3, Figure S3); thus proteins with sufficiently long amino acid sequences were input in seven sequence forms and considered an MBOMP if any of those were predicted positive. The lengths of 150 and 450 were chosen to cover the size of most $\beta$-barrel domains in BOMPs of known structure.

In the secondary structure prediction and manual inspection step, we disregarded any proteins or segments with more than $10 \% \alpha$-helical and less than $25 \%$ $\beta$-strand predicted secondary structure content and with the annotation of subcellular localization, family and domain, respectively.

\section{Sequence Logos}

For each ortholog set, we perfomed multiple alignment with ClustalW [57] and extracted the octomer region corresponding to the $\beta$-signal region proposed by Kutik et al [35]. From this set we removed identical octomers, yielding 57 octomers from the original 70 and 41 octomers from the expression confirmed set. We designed this procedure to trade off the desire to use as much data as possible, with the risk of creating biased results by including too many variations of highly similar sequences. Since the sequences are not all mutually independent, the heights of the columns are only suggestive, but the general trends remain the same when a sequence identity cutoff of $30 \%$ is employed to reduce sequence interdependence (Additional file 4, Figure S4).

\section{Additional material}

Additional file 1: Figure S1 - Multiple alignment of N-terminal region of putative Sam50 proteins. Multiple alignment and secondary structure prediction (by PSIPRED; orange: $\alpha$-sheet, blue: $\beta$-sheet) of the $\mathrm{N}$-terminal region of 9 proteins containing the bacterial surface antigen (POTRA) domain are shown.

Additional file 2: Figure S2 - $\beta$-signal motif match in YJL217W. (a) Secondary structure prediction (by PSIPRED) of the uncharacterized protein YJL217W and its match to the $\beta$-signal motif is shown. (b) $3 \mathrm{D}$ structure of YJL217W (PDBID:3012).

Additional file 3: Figure S3 - Sequence segmentation. The sequence segmentation used to search for, possible multiple domain, yeast MBOMPs with our SVM-predictor is shown.

Additional file 4: Figure S4 - Sequence logo of $\beta$-signal. Sequence logos are displayed for 70 MBOMP homologs which remain after omitting redundant sequences with more than $30 \%$ identity.

\section{Acknowledgements}

We thank Toshiya Endo and Koji Yamano for numerous fruitful discussions regarding MBOMPs and for pointing out the Kopec et al. results regarding Mmm2. We thank Kentaro Tomii for advice regarding FORTE. We thank the anonymous reviewers for suggestions which significantly improved this work. This research was partially supported by a Japan-Sweden Multidisciplinary BIO grant from the Japan Science and Technology Agency (JST). K.I. was supported as a research fellow of the Japanese Society for the Promotion of Science (JSPS).

\section{Author details}

${ }^{1}$ AIST, Computational Biology Research Center, Tokyo, Aomi, 135-0064, Japan. 2Japan Society for the Promotion of Science, Tokyo, Chiyoda, 102-8472, Japan. ${ }^{3}$ Taiho Pharmaceutical Company, Ibaraki, Tsukuba, 300-2611, Japan.

\section{Authors' contributions}

$\mathrm{KI}$ performed most of the experiments and prepared much of the manuscript. KI, NF and PH developed the novel MPOMP predictor. MG helped plan the study and refine the manuscript. PH conceived the study and wrote some of the manuscript. All authors read and approved the final manuscript.

Received: 10 September 2010 Accepted: 28 January 2011 Published: 28 January 2011

\section{References}

1. Sagan L: On the origin of mitosing cells. J Theor Biol 1967, 14(3):255-274

2. Kurland C, Andersson S: Origin and evolution of the mitochondrial proteome. Microbiol Mol Biol Rev 2000, 64(4):786-820.

3. Szafron D, Lu P, Greiner R, Wishart D, Poulin B, Eisner R, Lu Z, Anvik J, Macdonell C, Fyshe A, Meeuwis D: Proteome Analyst: custom predictions with explanations in a web-based tool for high-throughput 
proteome annotations. Nucleic Acids Res 2004, 32 Web Server: W365-371.

4. Walther DM, Rapaport D, Tommassen J: Biogenesis of beta-barrel membrane proteins in bacteria and eukaryotes: evolutionary conservation and divergence. Cell Mol Life Sci 2009, 66(17):2789-2804.

5. Baker KP, Schaniel A, Vestweber D, Schatz G: A yeast mitochondrial outer membrane protein essential for protein import and cell viability. Nature 1990, 348(6302):605-609.

6. Hill K, Model K, Ryan MT, Dietmeier K, Martin F, Wagner R, Pfanner N: Tom40 forms the hydrophilic channel of the mitochondrial import pore for preproteins. Nature 1998, 395(6701):516-521.

7. Wiedemann N, Kozjak V, Chacinska A, Schonfisch B, Rospert S, Ryan MT, Pfanner N, Meisinger C: Machinery for protein sorting and assembly in the mitochondrial outer membrane. Nature 2003, 424(6948):565-571.

8. Paschen SA, Waizenegger T, Stan T, Preuss M, Cyrklaff M, Hell K, Rapaport D, Neupert W: Evolutionary conservation of biogenesis of beta-barrel membrane proteins. Nature 2003, 426(6968):862-866.

9. Kozjak V, Wiedemann N, Milenkovic D, Lohaus C, Meyer HE, Guiard B, Meisinger C, Pfanner N: An essential role of Sam50 in the protein sorting and assembly machinery of the mitochondrial outer membrane. J Biol Chem 2003, 278(49):48520-48523.

10. Gentle I, Gabriel K, Beech P, Waller R, Lithgow T: The Omp85 family of proteins is essential for outer membrane biogenesis in mitochondria and bacteria. J Cell Biol 2004, 164:19-24.

11. Moslavac S, Mirus O, Bredemeier R, Soll J, von Haeseler A, Schleiff E: Conserved pore-forming regions in polypeptide-transporting proteins. FEBS J 2005, 272(6):1367-1378.

12. Dolezal P, Likic V, Tachezy J, Lithgow T: Evolution of the molecular machines for protein import into mitochondria. Science 2006, 313(5785):314-318.

13. Colombini $\mathrm{M}$ : A candidate for the permeability pathway of the outer mitochondrial membrane. Nature 1979, 279(5714):643-645.

14. Colombini M: VDAC: the channel at the interface between mitochondria and the cytosol. Mol Cell Biochem 2004, 256-257(1-2):107-115.

15. Shimizu S, Narita $M$, Tsujimoto $Y$ : Bcl-2 family proteins regulate the release of apoptogenic cytochrome $\mathrm{c}$ by the mitochondrial channel VDAC. Nature 1999, 399(6735):483-487.

16. Vander Heiden MG, Li XX, Gottleib E, Hill RB, Thompson CB, Colombini M: $\mathrm{BCl}-\mathrm{xL}$ promotes the open con guration of the voltage-dependent anion channel and metabolite passage through the outer mitochondrial membrane. J Biol Chem 2001, 276(22):19414-19419.

17. Simamura E, Shimada H, Hatta T, Hirai K: Mitochondrial voltage-dependent anion channels (VDACs) as novel pharmacological targets for anti-cancer agents. J Bioenerg Biomembr 2008, 40(3):213-217.

18. Sogo LF, Yaffe MP: Regulation of mitochondrial morphology and inheritance by Mdm10p, a protein of the mitochondrial outer membrane. J Cell Biol 1994, 126(6):1361-1373.

19. Meisinger C, Rissler M, Chacinska A, Szklarz LK, Milenkovic D, Kozjak V, Schonfisch B, Lohaus C, Meyer HE, Yaffe MP, Guiard B, Wiedemann N, Pfanner $\mathrm{N}$ : The mitochondrial morphology protein $\mathrm{Mdm} 10$ functions in assembly of the preprotein translocase of the outer membrane. Dev Cell 2004, 7:61-71.

20. Yamano K, Tanaka-Yamano S, Endo T: Mdm10 as a dynamic constituent of the TOB/SAM complex directs coordinated assembly of Tom40. EMBO Rep 2010, 11(3):187-193.

21. Kornmann B, Currie E, Collins S, Schuldiner M, Nunnari J, Weissman J, Walter P: An ER-mitochondria tethering complex revealed by a synthetic biology screen. Science 2009, 325(5939):477-481.

22. Youngman MJ, Hobbs AE, Burgess SM, Srinivasan M, Jensen RE: Mmm2p, a mitochondrial outer membrane protein required for yeast mitochondrial shape and maintenance of mtDNA nucleoids. J Cell Biol 2004, 164(5):677-688.

23. Imai K, Gromiha MM, Horton P: Mitochondrial $\beta$-Barrel Proteins, an Exclusive Club? Cell 2008, 135(7):1158-1159.

24. Kopec KO, Alva V, Lupas AN: Homology of SMP domains to the TULIP superfamily of lipid-binding proteins provides a structural basis for lipid exchange between ER and mitochondria. Bioinformatics 2010, 26(16):1927-1931.

25. Neupert W, Herrmann JM: Translocation of proteins into mitochondria. Annu Rev Biochem 2007, 76:723-749.

26. Endo T, Yamano K: Transport of proteins across or into the mitochondrial outer membrane. Biochimica et Biophysica Acta 2010, 1803(6):706-714.
27. Hiller S, Garces R, Malia T, Orekhov V, Colombini M, Wagner G: Solution structure of the integral human membrane protein VDAC-1 in detergent micelles. Science 2008, 321(5893):1206-1210.

28. Bayrhuber M, Meins T, Habeck M, Becker S, Giller K, Villinger $S$, Vonrhein C, Griesinger C, Zweckstetter M, Zeth K: Structure of the human voltage-dependent anion channel. Proc Natl Acad Sci 2008 105(40):15370-15375.

29. Ujwal R, Cascio D, Colletier JP, Faham S, Zhang J, Toro L, Ping P Abramson J: The crystal structure of mouse VDAC1 at $2.3 \mathrm{~A}$ resolution reveals mechanistic insights into metabolite gating. Proc Natl Acad SCi USA 2008, 105(46):17742-17747.

30. Colombini M: The published $3 \mathrm{D}$ structure of the VDAC channel: native or not? Trends Biochem Sci 2009, 34(8):382-389.

31. Zeth K: Structure and evolution of mitochondrial outer membrane proteins of beta-barrel topology. Biochim Biophys Acta 2010, 1797(6-7):1292-1299.

32. Zahedi RP, Sickmann A, Boehm AM, Winkler C, Zufall N, Schonfisch B, Guiard B, Pfanner N, Meisinger C: Proteomic analysis of the yeast mitochondrial outer membrane reveals accumulation of a subclass of preproteins. Mol Biol Cell 2006, 17(3):1436-1450.

33. Burri L, Vascotto K, Gentle IE, Chan NC, Beilharz T, Stapleton DI, Ramage L, Lithgow T: Integral membrane proteins in the mitochondrial outer membrane of Saccharomyces cerevisiae. FEBS J 2006, 273(7):1507-1515.

34. Remmert M, Linke D, Lupas AN, Soding J: HHomp-prediction and classification of outer membrane proteins. Nucleic Acids Res 2009, , 37 Web Server: W446-W451.

35. Kutik S, Stojanovski D, Becker L, Becker T, Meinecke M, Krüger V, Prinz C Meisinger C, Guiard B, Wagner R, Pfanner N, Wiedemann N: Dissecting membrane insertion of mitochondrial $\beta$-barrel proteins. Cell 2008, 132(6):1011-1024.

36. Walther D, Papic D, Bos M, Tommassen J, Rapaport D: Signals in bacterial $\beta$-barrel proteins are functional in eukaryotic cells for targeting to and assembly in mitochondria. Proc Natl Acad Sci 2009, 106(8):2531-2536.

37. The UniProt Consortium: The Universal Protein Resource (UniProt) in 2010. Nucleic Acids Res 2010, 38 Database: D142-D148.

38. Ashburner M, Ball CA, Blake JA, Botstein D, Butler H, Cherry JM, Davis AP, Dolinski K, Dwight SS, Eppig JT, Harris MA, Hill DP, Issel-Tarver L, Kasarskis A, Lewis S, Matese JC, Richardson JE, Ringwald M, Rubin GM, Sherlock G: Gene Ontology: tool for the unification of biology. Nature Genetics 2000, 25:25-29.

39. Schneider T, Stephens R: Sequence Logos: A New Way to Display Consensus Sequences. Nucleic Acids Res 1990, 18(20):6097-6100.

40. Crooks G, Hon G, Chandonia J, Brenner S: WebLogo: a sequence logo generator. Genome Research 2004, 14(6):1188-1190.

41. Kutik S, Stojanovski D, Becker T, Stroud D, Becker L, Meinecke M, Krüuger V, Prinz C, Meisinger C, Guiard B, Wagner R, Meisinger C, Pfanner N, Wiedemann N: The mitochondrial $\beta$-signal and protein sorting. Cell 2008, 135(7):1159-1160.

42. Gromiha $M$, Ponnuswamy P: Prediction of protein secondary structures from their hydrophobic characteristics. Int J Pept Protein Res 1995, 45(3):225-240.

43. Hunter S, Apweiler R, Attwood TK, Bairoch A, Bateman A, Binns D, Bork P, Das U, Daugherty L, Duquenne L, Finn RD, Gough J, Haft D, Hulo N, Kahn D, Kelly E, Laugraud A, Letunic I, Lonsdale D, Lopez R, Madera M, Maslen J, McAnulla C, McDowall J, Mistry J, Mitchell A, Mulder N, Natale D, Orengo C, Quinn AF, Selengut JD, Sigrist CJ, Thimma M, Thomas PD, Valentin F, Wilson D, Wu CH, Yeats C: InterPro: the integrative protein signature database. Nucleic Acids Res 2009, , 37 Database: D211-D215.

44. Kim S, Malinverni JC, Sliz P, Silhavy TJ, Harrison SC, Kahne D: Structure and function of an essential component of the outer membrane protein assembly machine. Science 2007, 317(5840):961-964.

45. Arnold T, Zeth $K$, Linke D: Omp85 from the thermophilic cyanobacterium Thermosynechococcus elongatus differs from proteobacterial Omp85 in structure and domain composition. J Biol Chem 2010, 285(23):18003-18015.

46. McGuffin L, Bryson K, Jones D: The PSIPRED protein structure prediction server. Bioinformatics 2000, 16:404-405.

47. Huh WK, Falvo JV, Gerke LC, Carroll AS, Howson RW, Weissman JS, O'Shea EK: Global analysis of protein localization in budding yeast. Nature 2003, 425(6959):686-691.

48. Schleiff E, Eichacker LA, Eckart K, Becker T, Mirus O, Stahl T, Soll J: Prediction of the plant beta-barrel proteome: a case study of the chloroplast outer envelope. Protein Sci 2003, 12(4):748-759. 
49. Soding J, Biegert A, Lupas AN: The HHpred interactive server for protein homology detection and structure prediction. Nucleic Acids Res 2005, 33 Web Server: W244-W248.

50. Tomii K, Akiyama Y: FORTE: a profile-profile comparison tool for protein fold recognition. Bioinformatics 2004, 20(4):594-595.

51. Wimley W: Toward genomic identification of beta-barrel membrane proteins: composition and architecture of known structures. Protein Sci 2002, 11(2):301-312.

52. Remmert M, Biegert A, Linke D, Lupas A, Söding J: Evolution of outer membrane $\beta$-barrels from and ancestral $\beta \beta$-hairpin. Molecular Biology and Evolution 2010, 27(6):1348-1358.

53. Koronakis V, Sharff A, Koronakis E, Luisi B, Hughes C: Crystal structure of the bacterial membrane protein TolC central to multidrug efflux and protein export. Nature 2000, 405(6789):914-919.

54. Velours G, Boucheron C, Manon S, Camougrand N: Dual cell wall/ mitochondria localization of the 'SUN' family proteins. FEMS Microbiol Lett 2002, 207(2):165-172.

55. Robert V, Volokhina EB, Senf F, Bos MP, Van Gelder P, Tommassen J: Assembly factor Omp85 recognizes its outer membrane protein substrates by a species-specific C-terminal motif. PLoS Biol 2006, 4(11) e377.

56. Boeckmann B, Bairoch A, Apweiler R, Blatter M-CAE, Gasteiger E, Martin MJ KM, O'Donovan C, Phan I, Pilbout S, Schneider M: The Swiss-Prot Protein Knowledgebase and its supplement TrEMBL in 2003. Nucleic Acids Res 2003, 31:365-370.

57. Thompson JD, Higgins DG, Gibson TJ: CLUSTAL W: improving the sensitivity of progressive multiple sequence alignment through sequence weighting, position-specific gap penalties and weight matrix choice. Nucleic Acids Res 1994, 22(22):4673-4680.

58. Kall L, Krogh A, Sonnhammer EL: A combined transmembrane topology and signal peptide prediction method. J Mol Biol 2004, 338(5):1027-1036

59. Krogh A, Larsson B, von Heijne G, Sonnhammer EL: Predicting transmembrane protein topology with a hidden Markov model: application to complete genomes. J Mol Biol 2001, 305(3):567-580.

60. Emanuelsson O, Nielsen H, Brunak S, von Heijne G: Predicting subcellular localization of proteins based on their $\mathrm{N}$-terminal amino acid sequence. J Mol Biol 2000, 300(4):1005-1016

61. Small I, Peeters N, Legeai F, Lurin C: Predotar: A tool for rapidly screening proteomes for $\mathrm{N}$-terminal targeting sequences. Proteomics 2004, 4(6):1581-1590.

62. Finn RD, Mistry J, Tate J, Coggill P, Heger A, Pollington JE, Gavin OL, Gunasekaran P, Ceric G, Forslund K, Holm L, Sonnhammer EL, Eddy SR, Bateman A: The Pfam protein families database. Nucleic Acids Res 2010, 38 Database: D211-D222.

63. Tusnády G, Dosztányi Z, Simon I: PDB_TM: selection and membrane localization of transmembrane proteins in the protein data bank. Nucleic Acids Res 2005, , 33 Database: D257-278.

64. Chou P, Fasman G: Prediction of the secondary structure of proteins from their amino acid sequence. Adv Enzymol Relat Areas Mol Biol 1978, 47:45-148.

65. von Heijne G: Signal sequences. The limits of variation. J Mol Biol 1985, 184:99-105

66. Kyte J, Doolittle R: A simple method for displaying the hydropathic character of a protein. J Mol Biol 1982, 157:105-132.

67. Berman H, Westbrook J, Feng Z, Gilliland G, Bhat T, Weissig H, Shindyalov I, Bourne P: The Protein Data Bank. Nucleic Acids Res 2000, 28:235-242.

68. Karlin S, Altschul SF: Methods for assessing the statistical significance of molecular sequence features by using general scoring schemes. PNAS 1990, 87(6):2264-2268.

doi:10.1186/1471-2164-12-79

Cite this article as: Imai et al.: Eukaryote-wide sequence analysis of mitochondrial $\beta$-barrel outer membrane proteins. BMC Genomics 2011 $12: 79$.

\section{Submit your next manuscript to BioMed Central and take full advantage of:}

- Convenient online submission

- Thorough peer review

- No space constraints or color figure charges

- Immediate publication on acceptance

- Inclusion in PubMed, CAS, Scopus and Google Scholar

- Research which is freely available for redistribution

Submit your manuscript at www.biomedcentral.com/submit
Biomed Central 